\title{
Development of Systems for 3D Target/Organ Definitions and Contouring
}

\author{
By
}

Bo Han

\author{
A thesis submitted to \\ the Faculty of Graduate Studies and Research \\ in partial fulfilment of \\ the requirements for the degree of \\ Master of Computer Science \\ Ottawa-Carleton Institute for Computer Science \\ School of Computer Science \\ Carleton University \\ Ottawa, Ontario
}

August 1, 2004

(C) Copyright

2004, Bo Han 


$\begin{array}{ll}\begin{array}{l}\text { Library and } \\ \text { Archives Canada }\end{array} & \begin{array}{l}\text { Bibliothèque et } \\ \text { Archives Canada }\end{array} \\ \begin{array}{l}\text { Published Heritage } \\ \text { Branch }\end{array} & \begin{array}{l}\text { Direction du } \\ \text { Patrimoine de l'édition }\end{array} \\ \begin{array}{l}\text { 395 Wellington Street } \\ \text { Ottawa ON K1A 0N4 }\end{array} & \begin{array}{l}\text { 395, rue Wellington } \\ \text { Ottawa ON K1A ON4 } \\ \text { Canada }\end{array} \\ \end{array}$

Your file Votre référence ISBN: 0-612-97453-7

Ourfile Notre référence ISBN: 0-612-97453-7

The author has granted a nonexclusive license allowing the Library and Archives Canada to reproduce, loan, distribute or sell copies of this thesis in microform, paper or electronic formats.

The author retains ownership of the copyright in this thesis. Neither the thesis nor substantial extracts from it may be printed or otherwise reproduced without the author's permission.
L'auteur a accordé une licence non exclusive permettant à la Bibliothèque et Archives Canada de reproduire, prêter, distribuer ou vendre des copies de cette thèse sous la forme de microfiche/film, de reproduction sur papier ou sur format électronique.

L'auteur conserve la propriété du droit d'auteur qui protège cette thèse. $\mathrm{Ni}$ la thèse ni des extraits substantiels de celle-ci ne doivent être imprimés ou aturement reproduits sans son autorisation.
In compliance with the Canadian Privacy Act some supporting forms may have been removed from this thesis.

While these forms may be included in the document page count, their removal does not represent any loss of content from the thesis.
Conformément à la loi canadienne sur la protection de la vie privée, quelques formulaires secondaires ont été enlevés de cette thèse.

Bien que ces formulaires aient inclus dans la pagination, il n'y aura aucun contenu manquant. 
PACLRATION ERROR.

IEXT COMELETE.
ERREU DE RAGINAION.

LE IEXTE EST CONELET. 


\section{Abstract}

Prostate delineation has shown considerable variability among physicians due to the poor image quality of Computerized Tomography (CT). The objective of this thesis is to develop a system for accurate estimation of physicians' target definitions in both two-dimension (2D) and three-dimension (3D) domains. The thesis work also developed a contouring system for improving the prostate definitions by computing oblique CT slices using different viewing angles of the prostate. CT and Photo images used in this thesis are from the visible human project (VHP). Three physicians at the Ottawa Regional Cancer Center (ORCC) defined the prostate on CT slices and each physician defined the same target twenty times. A demo of the developed systems was given to some physicians at the ORCC. These physicians expressed great interest in the tools we developed. They also stated that they would like to have such a tool integrated into their current planning system. 


\section{Acknowledgements}

I am very grateful to my advisor, Professor Jörg-Rüdiger Sack, for giving me the opportunity to work in his exciting research group, for his financial support throughout my Masters years (funded by Sun Micro systems and NSERC), and for his invaluable guidance and encouragement in this thesis work. I am very grateful to Professor Doron Nussbaum, for keeping me on the right direction in the medical field, and for taking time to discuss problems which concerned me most, without his guidance and insight in the medical field I would have been lost.

I am thankful to Jeff Gao for the numerus discussions we had and the data sources he recommended. This work has benefited greatly from accessing to vast amounts of anatomical pelvic data from the Visible Human Project and prostate definition data from the ORCC, for which I acknowledge my thanks to Dr. Michael at the VHP, Dr. Libni, Dr. Lee at the ORCC for their support in granting permission to use their precious data. My thanks also goes to Katie Bryant at Carleton's writing tutorial service for correcting the grammar errors in my thesis writing, to Gao Hua, Young-Soo for their help in the Paradigm lab.

My family has been always my backbone of support. It has been a long journey for 
them to bear with me, with all their understanding and patience, during the course of this research.

Finally I would like thank the School of computer science, and Carleton University, for upholding the spirit of education and research. 


\section{Contents}

Abstract iii

Acknowledgements iv

1 Introduction 1

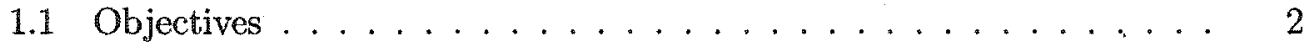

1.2 Main contribution . . . . . . . . . . . . . . 3

1.3 Thesis organization $\ldots \ldots \ldots \ldots . \ldots \ldots$

2 Background 4

2.1 Surface reconstruction $\ldots \ldots \ldots \ldots$

2.2 Volume computation $\ldots \ldots \ldots \ldots \ldots \ldots$

2.3 Image registration $\ldots \ldots \ldots \ldots \ldots$

2.3.1 Component descriptions . . . . . . . . . . . . . 9

2.3 .2 Related work .................... 13

2.4 Evaluation statistics . . . . . . . . . . . . 15

2.5 Contouring tool ................... 17

2.6 System platform. . . . . . . . . . . . . . 19 
3 Research work 20

3.1 Surface reconstruction $\ldots \ldots \ldots \ldots . \ldots . \ldots . \ldots 20$

3.2 Volume between adjacent slices $\ldots \ldots \ldots . \ldots . \ldots 21$

3.2 .1 Algorithm . . . . . . . . . . . . . . . 22

3.2 .2 Triangle normal $\ldots \ldots \ldots \ldots \ldots \ldots$

3.2 .3 Tetrahedron volume $\ldots \ldots \ldots \ldots \ldots \ldots$

3.3 Image registration $\ldots \ldots \ldots \ldots . \ldots \ldots$

3.3 .1 Registration modules $\ldots \ldots \ldots \ldots \ldots$. . . . . . . 27

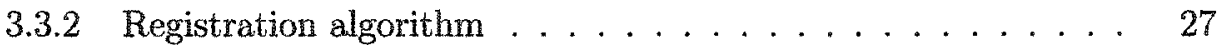

3.3.3 Multi-resolution registration . . . . . . . . . . . . . 29

3.4 Statistics . . . . . . . . . . . . . . . . . . . . 29

$3.4 .1 \mathrm{CT}$ and Photo volume $\ldots \ldots \ldots \ldots \ldots$

3.4 .2 Volume intersection and containment . . . . . . . . 30

3.4 .3 Gaps and directions of variations ............. 32

3.4 .4 Intra- and inter-physician variation $\ldots \ldots \ldots 33$

3.4 .5 Confidence level . . . . . . . . . . . . . . . 34

3.5 Oblique views of transverse definition . . . . . . . . 35

3.6 Contouring system $\ldots \ldots \ldots \ldots \ldots \ldots \ldots$

3.6.1 Oblique $\mathrm{CT}$ computation ................ 36

3.6 .2 Organ/target Contouring Process . . . . . . . . . . 42

3.6.3 Presenting the definition on the other views $\ldots \ldots . \ldots 45$

4 System development 49

4.1 Evaluation system $/$ tool . . . . . . . . . . . . . . 49

4.1 .1 Overview of physician evaluation system/tool ...... 50 
4.1 .2 Component design . . . . . . . . . . . . . 51

4.2 Contouring system $\ldots \ldots \ldots \ldots \ldots \ldots$

4.2 .1 System overview . . . . . . . . . . . . . 59

4.2 .2 Component design $\ldots \ldots \ldots \ldots . \ldots . \ldots 59$

4.3 Environment setup . . . . . . . . . . . . . . . 63

4.4 Issues of the contouring system $\ldots \ldots \ldots 64$

5 Experiments 65

5.1 Data source . . . . . . . . . . . . . . . . . 65

5.2 The evaluation system $\ldots \ldots \ldots \ldots \ldots \ldots$

5.2 .1 System operation . . . . . . . . . . . . 67

5.2 .2 Prostate reconstruction . . . . . . . . . . . . . . 69

$5.2 .3 \mathrm{CT}$ and Photo Registration $\ldots \ldots \ldots \ldots 71$

$5.2 .42 \mathrm{D}$ error analysis $\ldots \ldots \ldots \ldots \ldots \ldots$

$5.2 .53 \mathrm{D}$ error analysis $\ldots \ldots \ldots \ldots \ldots . \ldots \ldots$

5.2 .6 Oblique presentation of definitions $\ldots \ldots \ldots . \ldots 84$

5.3 Contouring system $\ldots \ldots \ldots \ldots$. . . . . . . . . . . 84

5.3 .1 System operation ................. 85

5.3 .2 The three window contouring $\ldots \ldots \ldots \ldots$

5.3 .3 Issue in computing oblique slices . . . . . . . . . 88

6 Conclusion 89

Bibliography 


\section{Chapter 1}

\section{Introduction}

Prostate cancer is one of the most common cancers in man organs. This type of cancers is typically confined to the prostate gland. It can be accurately treated if the prostate is identified. CT is a widely used method for diagnosing cancers and for planing radiation treatments for the cancers. Traditionally, Oncologists identify a prostate by drawing contours on a set of pelvic CT slices. But CT has poor image quality, which causes poor delineation of the prostate. The definition of the prostate is commonly done in a 2D style, slice by slice on a clinic planning system. The problem arises when a physician can not tell the prostate boundary apart from its surrounding soft-tissue. Then the physician has to estimate the cancer position, thus over- and under-estimations will occur. It is not only this but also that as a result, healthy tissue which is sensitive to radiation may be damaged and/or turn out all may not be radiated.

The problem of the over- and under-estimations inspired our idea that a physician 
requires a help tool other than the traditional $2 \mathrm{D}$ contouring tool. The help tool is to enable a physician to view the organ or the tumor from different angles, and may provide some extra surrounding structures, such that the physician could more precisely define the prostate. Oblique views are formed by cutting the prostate in a degree to the transverse direction.

\section{$1.1 \quad$ Objectives}

Objective 1: Help the physicians to evaluate the quality of the current prostate contouring method using CT images, and find out statistically, how much of the definition errors are due to poor CT image quality. Traditionally the methods for the quality evaluation are mostly in 2D and CT images are used as a gold standard for the error comparisons. This kind of evaluations are not very visual and also not very accurate. Our system is designed for both 2D and 3D evaluations, and Photo images are used as the gold standard.

In order to achieve the first objective, we design the evaluation system which consists of: a) constructing 3D CT prostate and 3D Photo prostate; b) obtaining a transformation mapping from CT to Photo (registration); c) transforming physicians' contours onto the Photo using the transformation mapping obtained in step b); d) computing statistical data from the two reconstructed objects; e) oblique presentation

The problem in identifying and contouring the target/organ (in our case, the prostate) leads us to the second objective:

Objective 2: Assist Oncologists to identify the prostate, by building a system using 
the oblique method, which has the abilities of manual contouring; contour editing; and demonstrating the contouring results on the views with different angles.

\subsection{Main contribution}

Our main contributions for this thesis are:

a. Development of

- an evaluation system to estimate the errors of physicians' CT definitions;

- implementation of Christiansen's 3D surface reconstruction algorithm [1];

- registration of CT and Photo images;

- statistics computation and oblique presentation of quality of contours;

- a new accurate and fast 3D volume computation algorithm;

- a contouring tool to assist the physicians to more precisely define the prostate;

b. Introduced a new contouring approach using oblique slices (3D contouring).

\subsection{Thesis organization}

The rest of this thesis is organized as follows: Chapter 2 presents the related work on 3D surface reconstruction, volume computation, 3D image registration, evaluation statistics, oblique presentation and the contouring tool. Chapter 3 and 4 discuss the contour evaluation system and 3D contour system respectively. Chapter 5 presents our experiment results of the systems. Chapter 6 gives the conclusion and future work. 


\section{Chapter 2}

\section{Background}

In this chapter, we present an overview of the research areas that are closely related to our work. We first discuss 3D surface reconstruction from parallel slices and prostate volume computation. Then we present 3D image registration using mutual information, the evaluation statistics, and the contouring system. We will introduce our system platform (Matlab).

\subsection{Surface reconstruction}

3D surface reconstruction (as studied here) is the ability to reconstruct a threedimensional structure from a sequence of parallel slices. In our case, a physician contours the prostate on a sequence of CT slices using a radiation planning system. Three dimensional reconstruction can be classified as volume-based reconstruction and surface-based reconstruction. Volume-based approaches $[2,3,4]$, require that the 
spacing between slices is comparable to the resolution within a slice. Surface-based approach is more widely used $[1,5,6,7,9]$. It assumes that the data defines the intersections of the object surface and slicing planes.

Which approach is more applicable depends on the nature of the data. When the available data are 3D grid values, a volume based approach like the marching cubes algorithm $[2,3]$ is suitable. If the available data are a set of closed contours with large separations between the adjacent slices, surface-based approach is suitable. We decided to use the surface-based approach, because the gaps between slices are $3 \mathrm{~mm}$, which is larger than the pixel size of $0.89 \mathrm{~mm}$ by $0.89 \mathrm{~mm}$ in a slice of our data.

Various of surface reconstruction methods have been published in literature. HownWen et al. [7] uses curvature samplings of contours to reduce contour points. The sampled points (the most curved points) are candidates for the control points of a Hermite interpolation which is essentially a cubic interpolation between four control points. One-to-one mapping based on the nearest-neighbor criterion is required between control points in a pair of adjacent contour slices. The sampled points without mapping need to be discarded and the discarding may cause shape deformation. Coleman et al. [8] fits each CT slice to a closed cubic b-spline curve and re-samples this b-spline so that each contour contains the same number of points, for the purpose of modeling a smoother surface. Zizzari et al. [9] and Jeoung et al. [5] proposed bi-parametric cubic B-spline methods to reconstruct rectangular-like surfaces with approximated and uniformly spaced contour points. One parametric B-spline is in contour direction, and the other is in longitudinal direction. B-spline points in each direction are re-sampled based on computed spline coefficients. Fuji et al. [6] introduced a deformation method which is good for terrain shape modeling. The algorithm 
first triangulates the area between two projected contours; then starts to eliminate the triangles one by one in an inward fashion until the outer contour fits with the inner contour. At the same time, the algorithm keeps track of the path-lines from the outer contour vertex to a point on the inner contour. The path-lines are used to construct a $3 \mathrm{D}$ shape of the object being examined. New points are introduced in the process of the path-lines' creation, which do not exist on the original contours. The complexity of the algorithm is $\Theta(n \log n)$ for the average case and $\Theta\left(n^{2}\right)$ for the worst case, where $n$ is the number of points on a contour. The algorithm assume that every two consecutive contours do not intersect each other. Fuji presented a heuristic scaling approach to overcome the limitation of the 'no-intersection requirement' with the cost of inflating or deflating the contours to make one contour contained within the other contour.

Christiansen et al. [1] uses the shortest diagonal algorithm for the surface reconstruction. In this algorithm, a surface is reconstructed by using tiles of triangles. That is the surface between each pair of adjacent slices is triangulated by connecting their contour points. In the reconstruction process, it is assumed that the data set consists of $m$ slices, each containing a slice contour. A contour on slice $i(1 \leq i \leq n)$ is a simple $n$-vertices polygon $p_{1}^{i} p_{2}^{i} \ldots p_{n}^{i}$, where $p_{1}^{i}, p_{2}^{i}, \ldots, p_{n}^{i}$ is an ordered sequence of vertices. In this algorithm, the initial two points of the triangulation are estimated, and we will describe how to estimate the initial points in our work in Chapter 3. Once the first line segment(formed by the initial two points) between the contours is established, the algorithm forms a $3 \mathrm{D}$ volume by generating a sequence of triangles as following: The vertices of each triangle are taken from the contour points. As shown in Figure 2.1 below, the triangle can be represented by its three vertices $p_{j}^{i}$, 
$p_{k}^{i+1}, p_{j+1}^{i}$, or $p_{j}^{i}, p_{k}^{i+1}, p_{k+1}^{i+1}$, where $p_{j}^{i}$ and $p_{j+1}^{i}$ are in the contour $i$ with the index $j$ $p_{k}^{i+1}$ and $p_{k+1}^{i+1}$ are in the contour $i+1$ with the index $k$. Depending on which of the diagonals of $p_{j}^{i} \rightarrow p_{k+1}^{i+1}$ and $p_{k}^{i+1} \rightarrow p_{j+1}^{i}$ is shorter, either the point $p_{j+1}^{i}$ or the point $p_{k+1}^{i+1}$ is chosen as the third point of the triangle. In this way, the third point is chosen iteratively by the shortest diagonal algorithm. The algorithm works for both convex case and non-convex case [1].

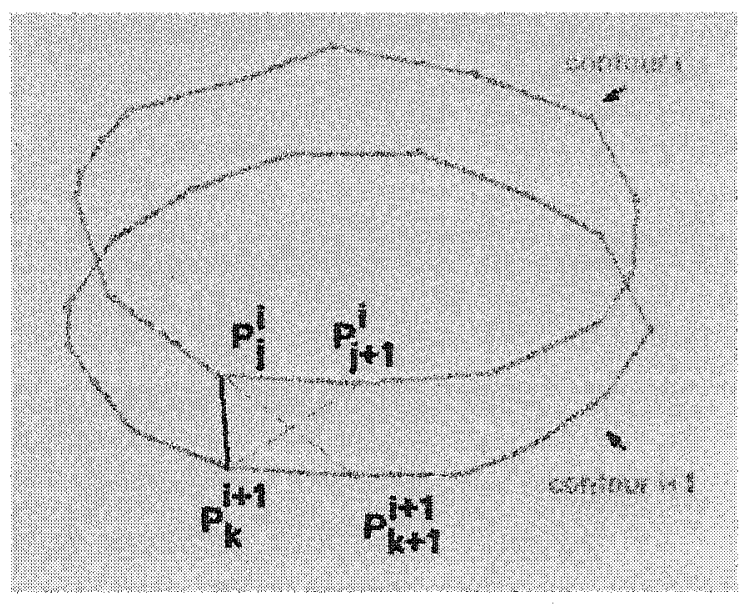

Figure 2.1: The triangulation by the shortest diagonal algorithm[1]

\subsection{Volume computation}

The volume computation is to compute the target volume from a set of parallel slices. In literature, target volume is commonly computed using some approximated methods. Ketting et al. $[30,31]$ derive target volume by multiplying the target area drawn on each slice by the slice thickness and then adding the contributions of all slices. This method can produce stepping-stair shaped target volumes and the 
variations of the volumes computed are significant when the slice thickness is large. Tai et al. [29] assume target volume can be represented by an ellipsoid, and the transverse and longitude diameters of the ellipsoid are used to compute the target volume. Formula 2.1 below is used by Tai et al. for obtaining the target volume:

$$
V=\frac{\pi}{6} \cdot d^{2} \cdot l
$$

where $V$ is the target volume, $d$ is the transverse diameter and $l$ is the longitude diameter. We think the ellipsoid approximation is not very accurate, because every target could have a different irregular shape. The difference is obvious when we look at the target contours of slices. Thus we design a new method to compute the prostate volume between each pair of adjacent slices, which will be described in detail in Chapter 3.

\subsection{Image registration}

Image registration techniques provide a common coordinate system between different sets of images. The techniques are used to map the images into a single coordinate system. In this research work, we will focus on the registration of different modalities. A registration program mainly consists of four components: transformation, interpolation, similarity measure and similarity optimization. Figure 2.2 gives an overview of the integration of the components. A description of the four registration components is given in Section 2.3.1. The related work in literature is presented in Section 2.3.2. 


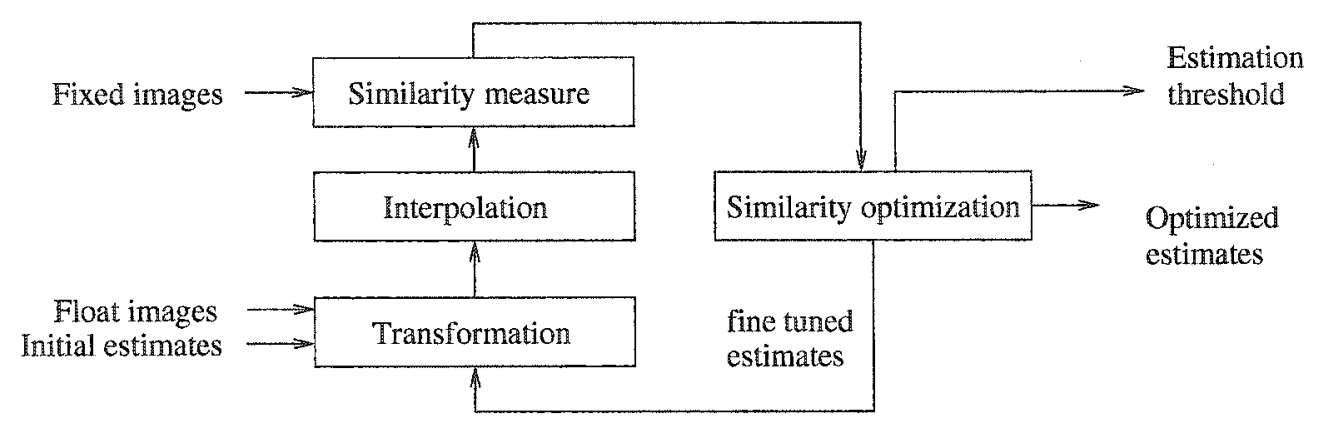

Figure 2.2: An overview of the integration of the registration components

\subsubsection{Component descriptions}

\section{Transformation}

In order to register two sets of images, a geometric transformation is needed, which is a linear function that takes a point and maps it into another point. Transformation can be classified into rigid, affine, projective and curved transformation. A 3D rigid transformation can be described using homogeneous $4 \times 4$ matrices as shown in Equation 2.2. Normally only one set of images (float) gets transformed and the other set of images (fixed) remains unchanged. Once the images (float) are transformed, interpolation techniques (described below) are used to obtain the intensity values in the transformed images.

$$
T=\left[\begin{array}{llll}
1 & 0 & 0 & t x \\
0 & 1 & 0 & t y \\
0 & 0 & 1 & t z \\
0 & 0 & 0 & 1
\end{array}\right]\left[\begin{array}{cccc}
1 & 0 & 0 & 0 \\
0 & \cos (r x) & -\sin (r x) & 0 \\
0 & \sin (r x) & \cos (r x) & 0 \\
0 & 0 & 0 & 1
\end{array}\right]\left[\begin{array}{cccc}
\cos (r y) & 0 & -\sin (r y) & 0 \\
0 & 1 & 0 & 0 \\
-\sin (r y) & 0 & \cos (r y) & 0 \\
0 & 0 & 0 & 1
\end{array}\right]\left[\begin{array}{cccc}
\cos (r z) & -\sin (r z) & 0 & 0 \\
\sin (r z) & \cos (r z) & 0 & 0 \\
0 & 0 & 1 & 0 \\
0 & 0 & 0 & 1
\end{array}\right]
$$

\section{Interpolation}

Interpolation is used to compute the grey values of the points which are the mapping 
from one image to another by the transformation. Interpolation methods can be classified as nearest neighbor, trilinear, cubic spline, and higher order nonlinear methods. When mapping an image (lloating) to another image (fixed), a newly mapped point will not necessarily be on a grid point. Thus interpolation is used to get the grey values of the grid points. After the interpolation, the similarities of the fixed image and the transformed float image are measured by some similarity measures which will be described below.

\section{Similarity measure}

A similarity measure is used to determine whether two sets of images are similar. The following paragraphs introduce the concept of relating the two sets of images using statistics of information, and the concept of measuring the statistics of information using mutual information.

Assume there are two medical image modalities of an anatomy, $A$ and $B$. The images of $A$ and $B$ have some differences in revealing the properties of the anatomy, that is they do not have the same values for the tissues in the images. In the statistical method, the anatomical volume is partitioned into a set of regions (e.g., the bones and the soft tissues). Each region shows an uniformed value with respect to the measurements of one kind of tissues, and each point in a region need not be spatially connected to the rest of the points with a same partition. The measurement value in $A$ is different with that in $B$ within a same region, e.g. for water, $A$ has value 0 , and $B$ has value 100 .

The set of regions in $A$ can be combined with that in $B$ and make a combined set of regions by intersecting the regions in the volumes. The combined regions can be 
delineated by both values of $A$ and $B$. We can use an intersected volume matrix to illustrate the combinations. The matrix rows represent the $A$ regions and the matrix columns represent the $B$ regions. Each entry value in the matrix represents the intersected volume between a region in $A$ and a region in $B$, and the corresponding region pair can be named a tissuevalue-pair.

The tissuevalue-pairs contain some statistical information with respect to the similarities between $A$ and $B$. The histogram for the tissuevalue-pair is used as the statistics of the combined volume. It represents the distribution of the tissuevaluepairs' occurrences in the combined volumes. A joint probability distribution of the two images can be obtained by dividing each intersected volume with the total volume.

It can be stated that the $A$ and the $B$ are similar if the tissuevalue-pairs of the two volumes occur many times (the occurrences are clustered) in the intersected volume. On the other hand, we say that they are dissimilar if few occurrences for the tissuevalue-pairs occur (the occurrences are scattered). Thus in this technique, we register the two modalities using the tissuevalue-pairs' occurrences, not the actual values between the two modalities in order to measure whether two volumes are similar. Therefore the similarities of $A$ and $B$ is related to the statistical information (tissuevalue-pairs' occurrences). The mutual information, which is based on the information theory, is used to measures the statistical dependence between two entities. The mutual information of $A$ and $B$ is defined as: $I(a, b)=\sum p(a, b) \log \frac{p(a, b)}{p(a) p(b)}$, where $p(a, b)$ is the join probability distribution of $A$ and $B ; a$ is the intensity variable of $A$; $b$ is the intensity variable of $B ; a$ and $b$ have fixed ranges (i.e. $0 \leq a, b \leq 255$ for grey 
images); $p(a)$ is the marginal probability distribution of $A ; p(b)$ is that of $B$. The values of $(a, b)$ represent the occurrences of the tissuevalue-pairs of the geometrical corresponding pixels in $A$ and $B$; The pixel values of $A$ and $B$ can be partitioned into different ranges, namely bins, for the similarity measurement. For example, the range of $[0,255]$ can be partitioned into 32 bins, 64 bins, 128 bins or 256 bins. A large number of bins can better characterize the anatomy than a small number of bins, but use more time in computing the similarity measurement. Normally 64 or 128 is considered a reasonable bin number. The counts (occurrences) of the tissuevaluepairs [13] are used to form a discrete histogram for the evaluation of the similarities of two modalities. Figure 2.3 gives an illustration of the tissuevalue-pairs and their bins on a 2D plot.

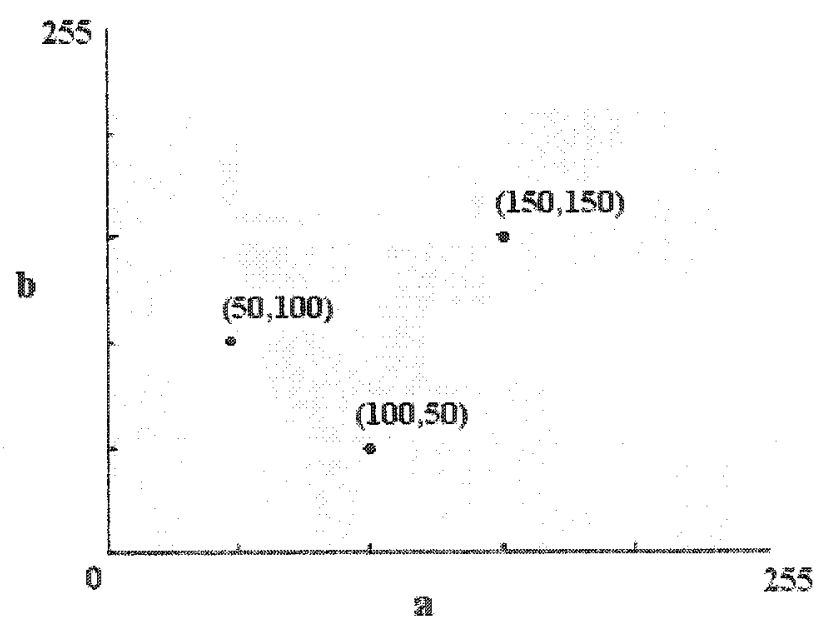

Figure 2.3: An illustration of the tissuevalue-pairs and their bins, where $a$ ( $x$-axis) is the intensity variable of $A ; b$ (y-axis) is the intensity variable of $B$; there are 256 bins for each axis, the three dots represent three tissuevalue-pairs and the dots' locations on the graph can be thought of as the bin baskets for storing the occurrences of the tissuevalue-pairs 


\section{Similarity optimization}

Image optimization methods find a global optimum similarity measurement by searching a set of transformation parameters in the parameter space. Popular methods in literature are Powell's line minimization, Steepest gradient descent, Downhill Simplex, Quasi-Newton/inverse Hessian, and Levenberg-Marquardt' least-squares minimization methods $[14,18]$.

Parameter optimization is a graduate and iterative process. The inputs to the optimization algorithm are a set of initial estimates of rotational parameters and translational parameters, which are rough guesses by knowledge or obtained by visual inspections. And the outputs are the best estimates within a preset threshold (e.g., $\left.10^{-3}\right)$.

\subsubsection{Related work}

Medical image registration algorithms can be classified as frame-based, point landmark based and voxel-based [17]. Frame-based methods are very accurate, but inconvenient as some frame is mounted on patient's body and hard to be placed in the same location the next time. Anatomical point landmark based methods are labor intensive and the accuracy depends on the precision in locating the corresponding anatomical points in the modalities. Voxel-based registration methods optimize some similarity measurement and measure the images' similarities by using all geometrically corresponding pixel pairs. The advantage is that all the intensity values of the images are used, no concerning of image segmentation. Woods et al. [16] uses 
a voxel-based method to register MRI and PET images, and assumes that the intensity values in different modalities are somehow correlated. Image segmentations are required in the registration to meet the assumption. Collignon et al. [12] first proposed the idea of using the mutual information (MT) for multi-modality image registration, and the idea has been extended and developed in many literature papers $[10,11,13,15,20,19]$. The advantage of using $\mathrm{MI}$ as the similarity measure is that the imaging modalities being registered are independent; the registration process can be automated, and there are no segmentations, no pre-processing and no assumptions on pixel values. The disadvantage of $\mathrm{MI}$ is that the size of overlapping part of two images influences the mutual information measure. When the overlap decreases, the MI may actually show an increase, which contradicts the assumption that if there is more overlap there is more mutual information. Studholme [11] introduced an overlap invariant MI measure to reduce the MI's sensitivities to overlap size, which is also called normalized mutual information (NMI). The NMI is defined in Formula 2.3:

$$
N M I(a, b)=\frac{\sum p(a) \log (p(a))+\sum p(b) \log (p(b))}{\sum p(a, b) \log (p(a, b))}
$$

where $a$ and $b$ are the intensity variables; $p(a, b)$ is the join probability distribution; $p(a)$ and $p(b)$ are the marginal probability distributions. Maes [13] proposed a trilinear partial volume distribution interpolation in mutual information image registration. This approach avoids introducing new intensity values which are not present in the original image. Therefore the partial volume interpolation technique is used to create joint histograms of intensities of the modalities. Figure 2.4 is a graphical illustration of partial volume interpolation in $2 \mathrm{D}$. 


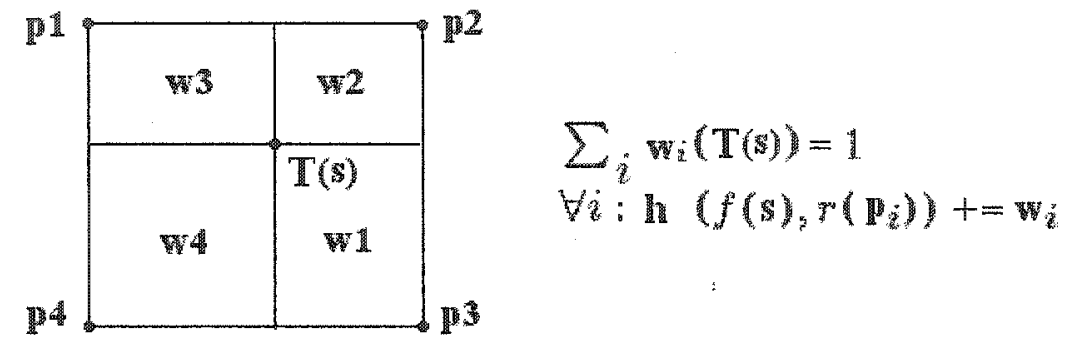

Figure 2.4: 2D illustration of partial volume trilinear interpolation, where $f$ is the float image; $r$ is the fixed image; $p 1, p 2, p 3, p 4$ are the grid points in the fixed image; $s$ is a point in the float image; $T(s)$ is the transformed point of $\mathrm{s}$ by the transformation $T ; w 1, w 2, w 3, w 4$ are the weights of $T(s)$ and $\sum w_{i}(T(s))=1 ; h$ is the joint histogram of $f$ and $r, f(s)$ is the grey value at $s$ in $f$, and $r(p)$ is the grey value at $p$ in $r$.

We will choose Maes's and Studholme's approaches in our registration program, and the details will be presented in Chapter 3 .

\subsection{Evaluation statistics}

Evaluation statistics are used for the quantitative evaluation of the physicians' target definitions. There is a large number of papers in literature which discuss the error analysis of target definitions on the CT by physicians [25, 24, 23, 29, 30, 31, 28, 26, 27]. In some papers, several experienced physicians define targets on patients' $\mathrm{CT}$, and use the CT definitions as a gold standard. Whereas some authors directly compare individual physician's definitions with those of other physicians involved, and measure the intra- and inter-physician variations. The commonly used evaluation statistics are the minimum, maximum, mean and standard deviation (3MSTD) of the definition 
coverage, the variation gaps, and the variation directions. The measurements of volume, surface area and radius are used for quantitative analysis in various literature papers. Cazzaniga et al. [30] and Ketting et al. [31] estimate the variations by comparing the definitions between four physicians involved. The standard deviations and the square root of the average variance (volume and radius) are used for the variation estimates of the lung and nasopharyngeal cancers. Tai et al. [36] uses the CT as the gold standard for the error analysis of the target definitions in his transCanada survey across Canada. In the survey, the physicians were asked to define the cervical esophageal cancer on a set of CT slices. The mean difference of the definition radii was used to measure intra- and inter-physician variations. The radii of the definitions were measured in eight different angles (e.g., anterior-posterior, lateral-lateral). Leunens [28] calculated the mean and the standard deviation of the target surface as the statistics in the assessment of the delineation for brain tumors. Only four angles were considered in the analysis of error directions. Fiorino [23] uses the mean contours drawn on the CT slices to estimate physicians' definition variability, and considers that the mean contours are more representative of interphysician errors. All of the statistics are only displayed in 2D. We will introduce some of the statistics that we designed differently, and also introduce a different way of viewing the target definitions in Chapter 3 . 


\subsection{Contouring tool}

Manual contouring tools are important components of clinical radiation treatment planning system (RTPS), in which the physicians define the targets by drawing contours on a sequence of CT slices. The CT contouring is done in a slice by slice style. Goitein [41] developed a multi-dimensional system which has the functionalities of the target volume delineation, the dose calculation and the therapy simulation. Goitein displays the transverse, the sagittal and the coronal target slices simultaneously on the system window. Transverse, sagittal, coronal planes are three orthogonal planes. Transverse plane (xy-plane) divides an object in a horizontal direction. Sagittal plane (yz-plane) divides an object into right and left halves. Whereas coronal plane (xzplane) divides an object into front and back portions. Figure 2.5 gives the three orthogonal views.

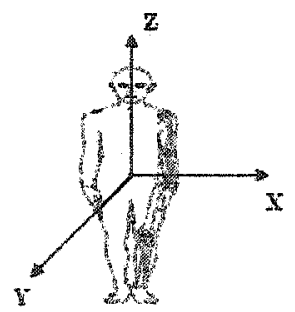

(a)

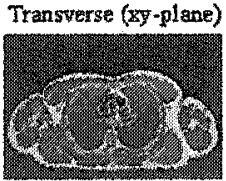

(ำ

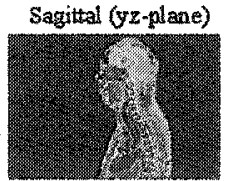

(c)

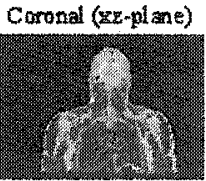

(al)

Figure 2.5: Images from three perspectives: transverse, sagittal, coronal (Courtesy of visiblehuman/atias). (a) shows a xyz coordinate system; (b) shows a transverse image (xy-direction) of buman body; (c) shows a sagittal image (yz-direction) of human body; (d) shows a coronal image (xz-direction) of human body.

The sagittal and the coronal slices are displayed for the cross-referencing during the data presentation and the target contouring. On the transverse slice, there are buttons 
for traversing through the target slices and there are colored pointers to indicate where the sagittal and coronal views are centered. On the sagittal and the coronal slices, there are lines to indicate where the current, next, last transverse slices are located. The system's console has the ability to zoom the image and to rapidly review a sequential of images. Goitein considers that displaying the three views together simultaneously helps the user to gain three dimensional information of the target. Segment drawing and contiguous drawing are featured in the system. In segment drawing mode, users can move the cursor to a desire position and start a segment drawing by pressing down the cursor and drag along. In contiguous drawing mode, users move the cursor around with a constantly updated line which connects the last selected point to the current cursor position. The points are selected by clicking the right mouse button while moving. The system enables the users to define the target and place annotations on the images. In Mohan's [40] 3D RTPS system, combination of manual and automatic contouring methods were proposed to delineate the target. Some of the contours may be drawn in automatic mode but users may need to guide the cursor manually through the low-contrast anatomy where the automatic method fails. The contours can be copied from one slice to the next in the sequence. Those contours that do not match the structure can be edited or redrawn. Zooming feature is also included to help outlining of small or critical regions.

In the systems reviewed above, the target contours are drawn only on the transverse slices and there are no designs to display the transverse contour definitions on the other two views. Users would appreciate it if the oblique views with any angles are added rather than only the sagittal and the coronal views. We will discuss our oblique techniques Chapter 3. 


\subsection{System platform}

Matlab is a scientific computing and model-based design software [35]. It is a tool for doing numerical computations with matrices and vectors and it includes a large number of toolboxes for signal processing, statistical analysis, image processing, etc.

In this study, Matlab is chosen as a platform to build our evaluation and contouring system. The advantages of using Matlab are: 1) It can easily manipulate vectors and matrices; 2) It has a set of plotting functions for 2D and 3D visualization; 3) It provides a set of high-level GUI commands for fast system design and implementation; 4) It has many nice computing, analyzing and optimizing functions such as det, hist and fminsearch; 5) It provides good on-line documentations.

It is worth mentioning that Matlab, different from $\mathrm{C} / \mathrm{C}++$, starts the array indexing with 1 and not 0 . To access the pixel intensity at the sixth row, the first column, and the third slice, of the volume matrix $m$, we write $m(6,1,3)$. Arrays in Matlab are column-major and can be represented as a long one-dimensional array. ones One can picture this as Matlab taking column by column from the multi-dimensional array and placing them below one another in the one-dimensional array, stating at slice one. 


\section{Chapter 3}

\section{Research work}

This chapter presents our research on the 3D surface reconstruction from the parallel slices, the volume computation, the 3D image registration using mutual information, the statistics for the evaluations of the physicians' prostate definitions, and the contouring system.

\subsection{Surface reconstruction}

For this research, we aim to choose a surface reconstruction method which is simple, fast and easy to implement. As for our data, we do not have irregular shaped contours, nor do we have a branching problem (since we only have one contour within each slice), and we do not want to eliminate any contour points. Therefore we chose Christiansen et al.'s the shortest diagonal algorithm [1] to reconstruct our target surface by using tiles of triangles. The algorithm has the requirement that two contours have similar 
shape, similar size and a common central point. The first requirement is met by our data. The requirement of similar size and a common central point is met, in our implementation, by the scaling and mapping techniques which are described in the paper.

In the algorithm, the initial two points are estimated and the author did not mention what the estimation would actually be. We choose the first initial point randomly from one contour, and compute the second initial point from the other contour prior to triangulation as follows: first the pair of the adjacent contours are centered and scaled to a unit square such that the resulting contours have a common center and a similar size; then the second initial point can be obtained by finding the point on the second contour which has the shortest distance to the randomly selected first initial point. This choice ensures that the segment formed by the two initial points does not intersect a contour because the points are in different slices. Once the initial two points are computed, the algorithm proceed to choose the third point iteratively to form triangles described in Section 2.1.

\subsection{Volume between adjacent slices}

To compute the volume of a prostate, given the surface-triangulated volume described in the previous section above, is to compute the volume in-between each pair of adjacent slices and to add them up. We developed a volume computation method, which is simple and fast. The method can be applied to convex contours as well as non-convex contours. 


\subsubsection{Algorithm}

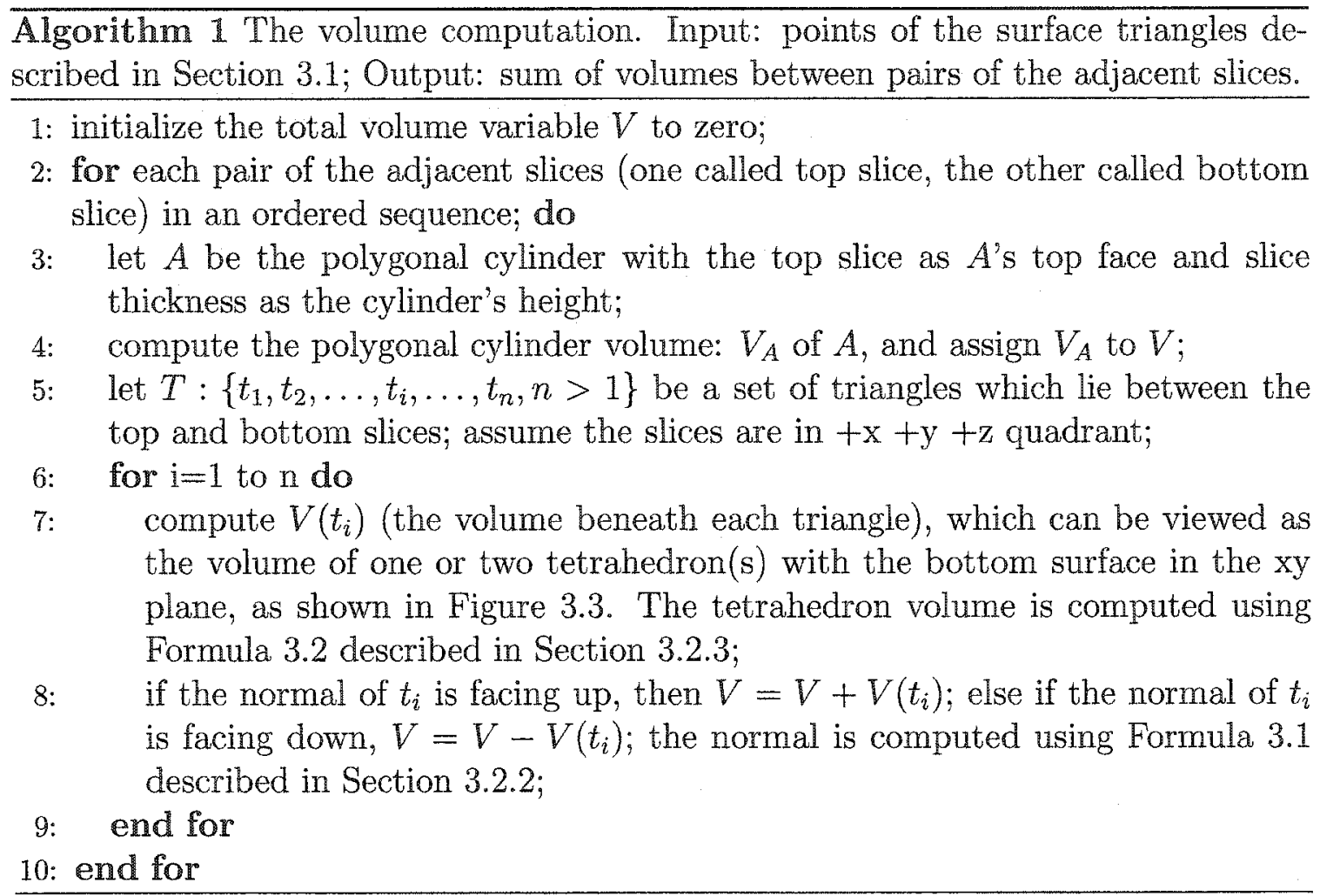

The complexity of Algorithm 1: $\Theta(n)$

The for loop from line 2 to line 10 iterates $\Theta(1)$ times because we only have constant number of the target slices (e.g., 13) and the contour of each slice contributes two times. Line 4 takes $\Theta(n)$ times, because the volume of a polygonal cylinder is the multiplication of the polygonal area by the cylinder high, and polygonal area can be computed using the equation: $A p=\frac{1}{2} \sum_{i=0}^{n-1}\left(x_{i} y_{i+1}-x_{i+1} y_{i}\right)$ [33], where $x, y$ are the coordinates of a point on a contour, $i$ is the index of the sequence of $n$ points. The normal and tetrahedron computation inside the second for loop (line 6 to line 9) takes $\Theta(1)$ based on the Formula 3.1 and 3.2 used. Therefore the cost of the second for loop is $\Theta(n)$ and the same for the algorithm. 


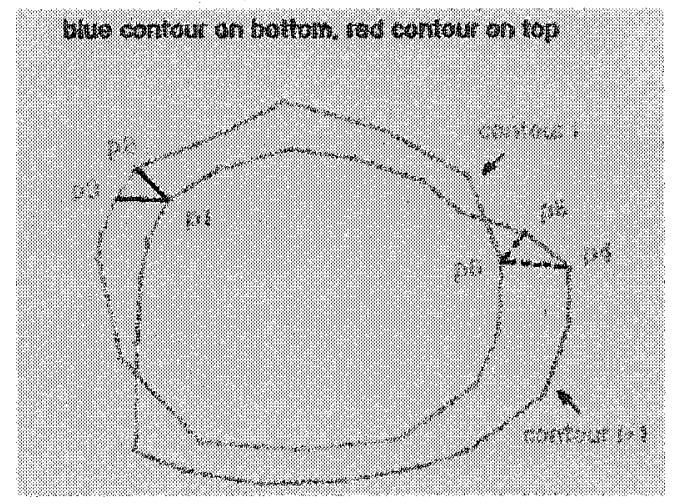

Figure 3.1: The normal of the $\Delta p 1 p 2 p 3$ is positive, thus the triangle on the left faces up; the normal of the $\Delta p 4 p 5 p 6$ is negative, thus the triangle on the right faces down.

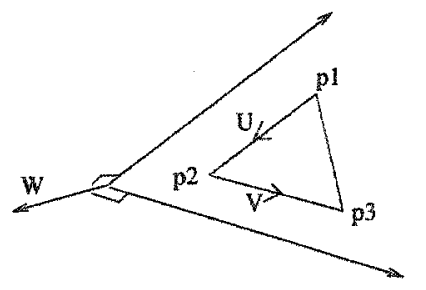

Figure 3.2: The normals of two vectors

\subsubsection{Triangle normal}

The orientation of a triangle is determined by the triangle's normal vector. The normal vector is the cross product of its two edge vectors(U,V), as seen in Figure 3.2. The cross product is a multiplication resulting in a third vector (triangle nomal) that is perpendicular to the plane of the two edge vectors. The order of the vector computation is counterclockwise. The cross-product direction is such that if one took his right hand and curled his fingers from $U^{-}$to $V$, his fingertips are on $V$ and his 
lower palm is on $U$, his thumb would point straight out in the direction of the third vector (triangle normal).

Let vector $\mathrm{i}$ be $(1,0,0)$, vector $\mathrm{j}$ be $(0,1,0)$, vector $\mathrm{k}$ be $(0,0,1) ; U\left(x_{u}, y_{u}, z_{u}\right)$ and $V\left(x_{v}, y_{v}, z_{v}\right)$ be the two vectors; then we can have Formula 3.1 below for the computation of the cross product [34]:

$$
W=\operatorname{cross}(U, V)=i\left(y_{u} z_{v}-y_{v} z_{u}\right)-j\left(x_{u} z_{v}-x_{v} z_{u}\right)+k\left(x_{u} y_{v}-x_{v} y_{u}\right)
$$

The triangle surface will have the orientation of facing up if the cross product is positive, and facing down if the cross product is negative. In other words, the direction of a triangle's normal vector decides whether the volume beneath the triangle should be added or deducted from the total volume.
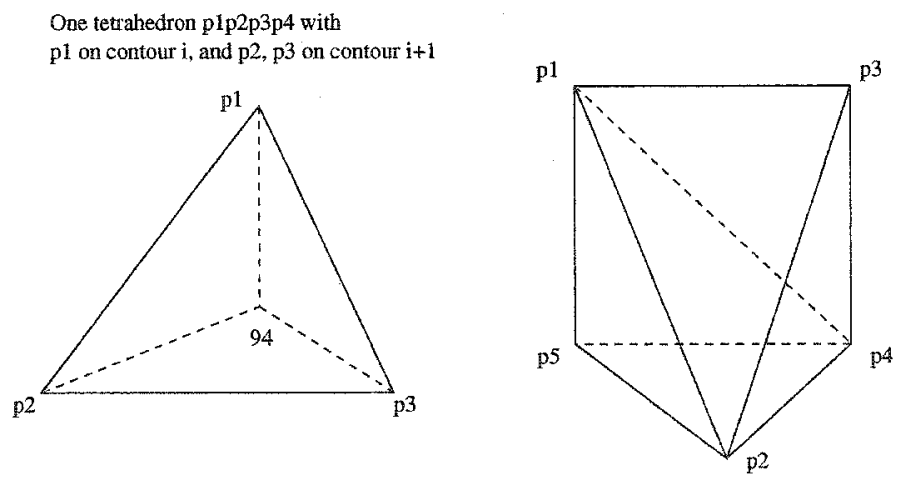

Two tetrahedra $\mathrm{p} 1 \mathrm{p} 2 \mathrm{p} 4 \mathrm{p} 5$ and $\mathrm{p} 1 \mathrm{p} 2 \mathrm{p} 4 \mathrm{p} 3$ with $p 1, p 3$ on contour $i$ and $p 2$ on contour $i+1$

Figure 3.3: The tetrahedra from two types of triangles 


\subsubsection{Tetrahedron volume}

The tetrahedron volume can be computed by using its six edges. The computation formula is presented in [32]. Let the six edge-lengths of the tetrahedron be $u, U, v$, $\mathrm{V}, \mathrm{w}, \mathrm{W}$, which are in any order that pairs opposite edges's lengths such that: $(\mathrm{u}, \mathrm{U})$, $(\mathrm{v}, \mathrm{V}),(\mathrm{w}, \mathrm{W})$ as shown in the Figure 3.4:

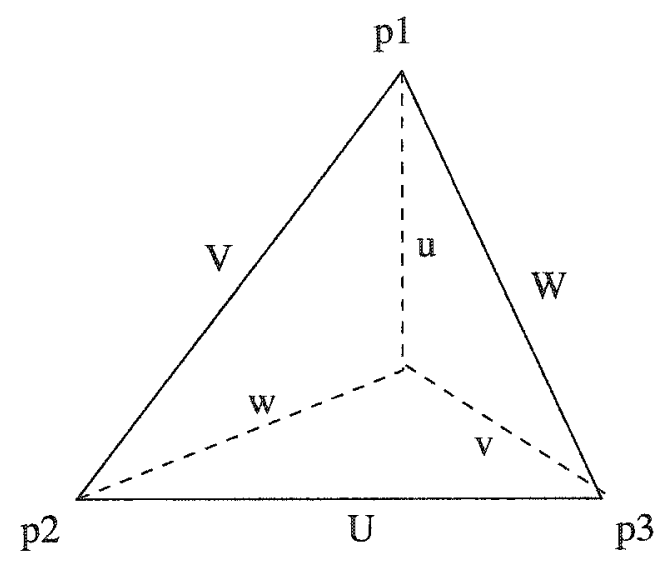

Figure 3.4: The tetrahedron with its six edge-lengths

Let $V$ be the volume of the tetrahedron, then we can have:

$$
V=\operatorname{sqrt}(\operatorname{det}(X) / 288)
$$

where $X$ is a matrix with the six edges of the tetrahedron, and det $(X)$ is the determinant of the matrix $X$. Below is the definition of the matrix $X$ [32]: 


$$
X=\left|\begin{array}{ccccc}
0 & u^{2} & v^{2} & w^{2} & 1 \\
u^{2} & 0 & W^{2} & V^{2} & 1 \\
v^{2} & W^{2} & 0 & U^{2} & 1 \\
w^{2} & V^{2} & U^{2} & 0 & 1 \\
1 & 1 & 1 & 1 & 0
\end{array}\right|
$$

and $\mathrm{X}$ can also be expressed as the following polynomial:

$X=\left(4 u^{2} v^{2} w^{2}-u^{2}\left(v^{2}+w^{2}-U^{2}\right)^{2}-v^{2}\left(w^{2}+u^{2}-V^{2}\right)^{2}-w^{2}\left(u^{2}+v^{2}-W^{2}\right)^{2}+\left(v^{2}+\right.\right.$ $\left.\left.\left.w^{2}-U^{2}\right) w^{2}+u^{2}-V^{2}\right)\left(u^{2}+v^{2}-W^{2}\right)\right) / 12$

\subsection{Image registration}

The mutual information method is chosen to register our CT and Photo images. We are not focusing on developing new algorithms, because image registration is a common practice and quite mature in the medical field. Instead, based on the literature review of the available algorithms for each module, we have chosen the algorithms that we consider the most appropriate for our system. The modules are

combined to form an automated registration unit in our 3D estimation system. In this system, we will use a rigid (no deformation), intensity based image registration method, because our CT and Photo datasets from VHP were acquired using a fresh and frozen cadaver and did not have any deformation in the data acquisition process. 


\subsubsection{Registration modules}

Our four registration modules are:

- transformation: 3D rigid, with 6 parameters: 3 rotations, and 3 translations on the $\mathrm{X}, \mathrm{Y}$, and $\mathrm{Z}$ axes;

- interpolation: trilinear partial volume distribution interpolation;

- similarity metric: mutual information;

- optimum search: minimization of mutual information (Levenberg Marquardt);

The assembled registration modules above have shown to be robust and accurate for the registration of the $3 \mathrm{D}$ pelvic images in our experiment. The experiment results will be shown in Chapter 5 .

\subsubsection{Registration algorithm}

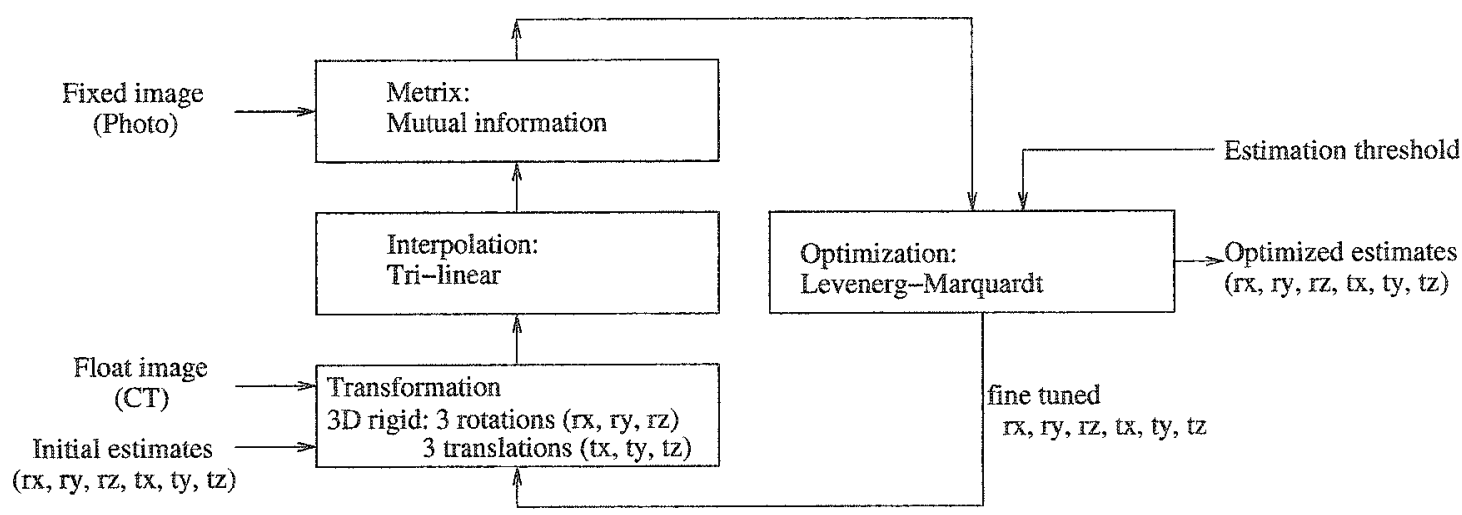

Figure 3.5: An overview of the $3 \mathrm{D}$ rigid registration system 


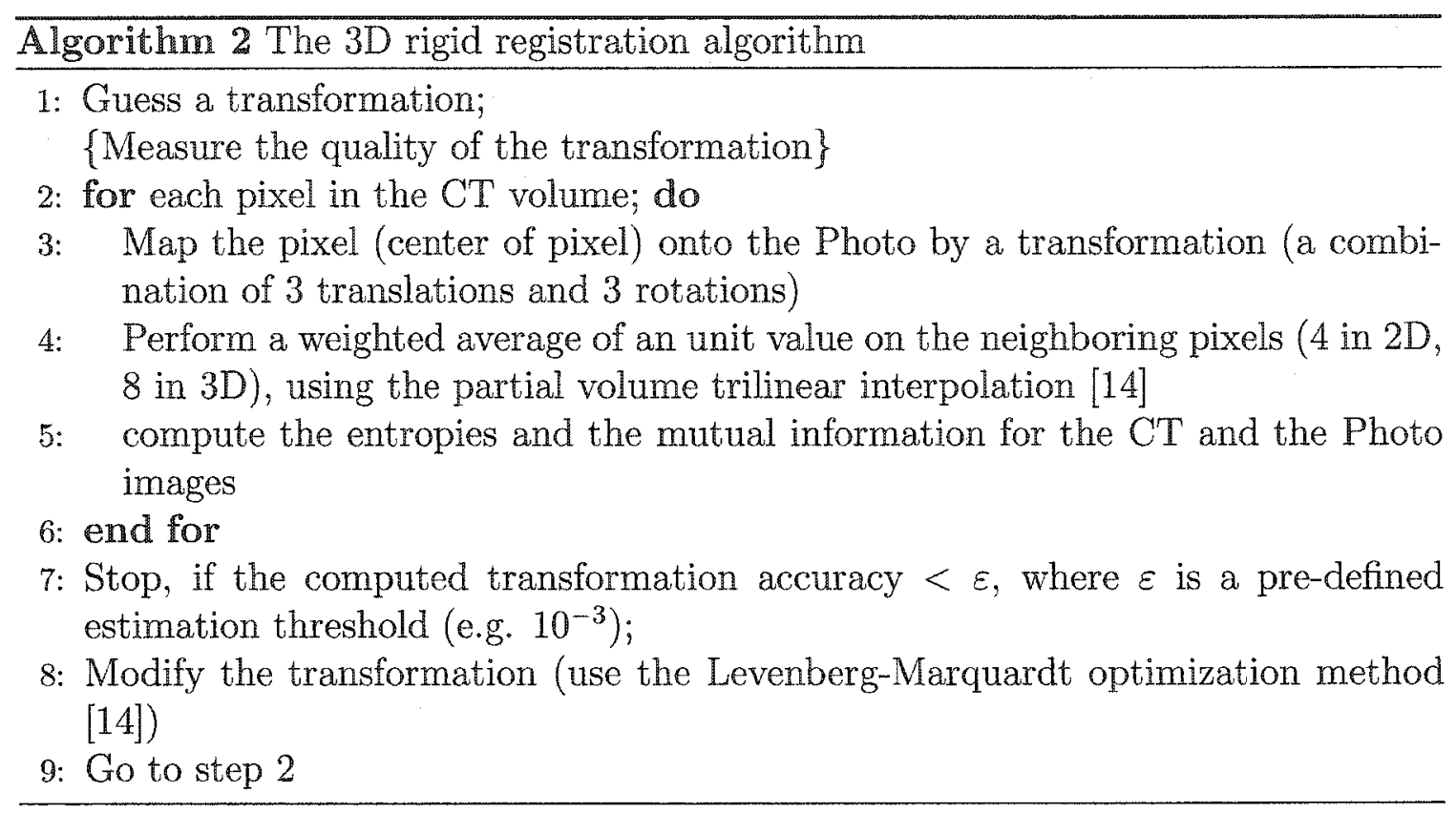

In our registration Algorithm 2, each pixel value corresponds to a bin location and the weighted values for the neighboring pixels are in the range of $\left[\begin{array}{ll}0 & 1\end{array}\right]$. If two images are registered, the joint histogram plot of the two modalities will show that the points on the histogram are more clustered; if not properly registered, the plot will show that the points on the histogram are more scattered.

The optimization steps ( $7 \& 8$ ) of Algorithm 2 are done by using Matlab's fminsearch function (an implementation of the Levenberg Marquardt's optimization algorithm). The estimation threshold can be set through the function's parameter options. Figure 3.5 gives an overview of how registration modules are integrated as a whole to perform the $3 \mathrm{D}$ rigid registration task. The registration accuracy of the $\mathrm{CT}$ and the Photo in our $3 \mathrm{D}$ system is measured by the checkerboard method, and the registration result is also presented to the physicians in our demostration given at the CCRA. 


\subsubsection{Multi-resolution registration}

In order to carry out a registration, we need to iteratively optimize the transformation estimates. This requires the iterative evaluations of the corresponding CT and Photo measurements (the joint probability distribution). This will be a very time consuming task for a large sized 3D full image registration. A multi-resolution technique (interpolation) is used to reduce the amount of evaluations. For the low resolution, a smaller number of measurement values of our CT and Photo images are used during the iterative evaluations. For example, a 3D full image registration of a size of $512 \times 512 \times 50$ would have been reduced by a factor of four if one out of two pixels in both the $\mathrm{X}$ and $\mathrm{Y}$ axes are picked in the process. When moving up to a higher resolution, the estimation parameters computed from the lower resolution stage (which is closer to the optimum parameters) are used.

\subsection{Statistics}

We have designed the algorithm for the 3D reconstruction of physicians' prostate definitions, and the algorithm for the registration of the CT and the Photo definitions onto a common coordinate system. It is time to compare and quantify the differences of the definitions for the two modalities, and to show how well the physicians' prostate estimations are under a poor CT imaging quality. We developed a set of measurements as the statistics for the evaluation of the physicians' prostate definitions, which includes the prostate volumes, the gap of difference, the directions of the over- and the under-estimation, and the physicians' confidence levels in the estimations. 


\subsubsection{CT and Photo volume}

The CT and the Photo volumes of the prostate will be computed by using the algorithm for the $3 \mathrm{D}$ reconstruction introduced in the previous chapter. We will compare the results of the CT and the Photo volumes using minimum, maximum and standard deviation (3MSTD) techniques. By using these techniques, we will see how much each of the CT definitions differs from those of the Photo's in terms of the volume amount. The average prostate volume would be around $30 \mathrm{~cm}^{3}(30 \mathrm{~g})$ and the size is similar to a walnut, based on the knowledge of the physicians. Our CT volumes from the three physicians' definitions will be constructed. We use the standard deviation to compare the definitions of the physicians. After the image registration, a 3D graphs will be plotted, in which the CT and the Photo volumes will be super-imposed onto one another and with different colors to get a transparent view of the volumes and their differences.

\subsubsection{Volume intersection and containment}

This measurement indicates how well the physicians' prostate definitions have covered our gold-standard definitions. Our inputs are the CT contours and the Photo contours of the prostate, and the output is the intersection of the two volumes. Because the volumes are formed by the parallel slices, our task is primarily to compute the intersection of the two corresponding slices of the CT and the Photo which are meant to be the same anatomical slice of the prostate in the two different modalities. Then we compute the intersected volume using the set of the intersected contours. The volume coverage will be the intersected volume over the Photo volume. We proposed 
the approximation approaches, which are the methods of the spline [9] and the regular line-intersection with rays from a median point, to approximate the intersected part of the two contours to be processed. Based on the comparison of the volumes enclosed under the triangulated-surface by the cubic spline and the regular-line methods, we consider that both approaches have fairly good approximation using the intersection points to replace the original contour points. The approaches are described as the following: a) the spline approach: model each contour as some cubic b-splines, which then form a closed contour; b) the regular line approach: model each contour as regular line segments, which then form a closed contour. For both approaches, first choose a centre point from those of the CT and the Photo slices. Then shoot from the centre point a set of rays which have evenly offset angles. There will be a set of new intersected points between the contours and the rays. For each ray, the closer intersecting point to the centre point will be chosen as our output point. After processing all of the ray lines, we obtain the approximated intersected portion of the $\mathrm{CT}$ contour and the Photo contour.

The Figure 3.6 illustrates how the intersected portion of two definitions are computed. One contour is the Photo definition, other is the CT definition. The closer point of the intersecting points with each ray is chosen and included in the set of the output points.

Time complexities for both regular-line and cubic spline methods are linear. The spline contours are more smooth, with the cost of additional time to compute the coefficients of the cubic splines. With $n$ contour points, we have $n-1$ segments of splines. Each spline has 4-coefficients to compute, totaling $4(n-1)$ coefficients. There are two end points for each spline, and their slopes have the combined constrains 


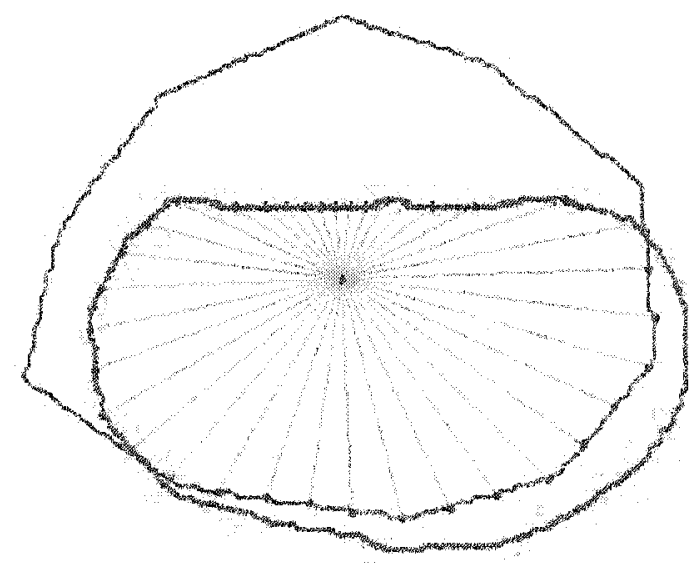

Figure 3.6: Intersected contour of the CT and the Gold

with their neighboring points. The spline method is linear, in terms of the number of coefficients [22].

\subsubsection{Gaps and directions of variations}

Using the above ray intersection methods, we can compute, for a particular slice, the minimum and the maximum gaps between the CT and the Photo definitions. These statistics will show the over-and under-estimations of the prostate definitions. We can choose a slice from the definitions of the physician(s) for the $2 \mathrm{D}$ evaluations, which has the minimum or the maximum error gap. Alternatively, we can choose a median gap slice for the evaluation. Since we are shooting the rays from the median point, with a set of evenly offset angles (like the bicycle's wheel rays), we can get the tendency directions of the over- and under-estimations of each slice as well. We define a positive gap as an over-estimate, and a negative gap as an under-estimate. 
A graph of the direction tendency will be plotted in our experiment. Based on the literature, the superior-inferior (front-back) and the anterior-posterior (top-bottom) positions have more definition variations than the lateral (left-right) positions, we will verify the position variations with our experiment results. We count the number of the slices which have been missed by the definitions or have been mistakenly included as part of the prostate for the gaps in the direction of the inferior-superior positions. The distance between a pair of the adjacent CT slices is $3 \mathrm{~mm}$.

\subsubsection{Intra- and inter-physician variation}

Intra-physician variations are to evaluate the self-difference (their own errors) of an individual physician in our study. On the other hand, the inter-physician variations are to compare the estimations between the physicians. The statistics of the 3MSTD will be computed. Since the median variation is more representative of the variabilities [23], we will include it in our statistics. It is worth considering that how the median definition among all of the definitions of the physician(s) is chosen. The large coverage rate will not necessarily mean a large contour area, and the small coverage will not necessarily mean a small contour area, due to all of the irregular contour shapes and the contour intersections. Therefore we proposed to compute a median definition by obtaining a median intersecting point along each ray, and connecting all of the median points to form our median contour. We consider this approach more logical. A 2D graph will be plotted in Chapter 5 to show the gap differences in a set of directions.

Figure 3.7 illustrates how median points are chosen from as set of the definitions for the same slice. We can overlay together all of the slice definitions by the physician(s) 


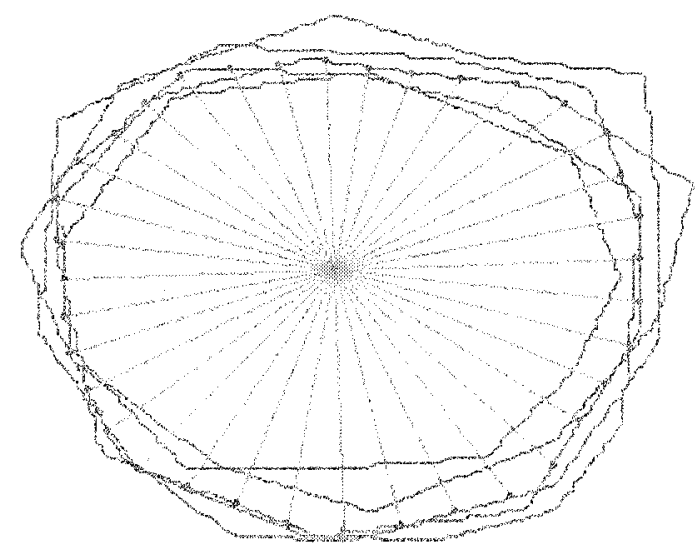

Figure 3.7: The median of a set of the definitions for a same slice

for the median computation.

\subsubsection{Confidence level}

This measurement shows our physicians' confidence levels in their prostate definitions of a slice, and to what extent they are more confident in their definitions. We overlay all definitions from one slice from a physician. For each pixel of the slice, we add up the number of times (frequency) it has been considered to be part of the prostate. We will plot a $2.5 \mathrm{D}$ surface graph to show the frequency results of the slice, with our Photo contour super-imposed on the surface graph in a different color. It can be imagined that at the center of the slice the graph will show a peak, and as we are moving away from the center, the graph will move downhill as the pixels being further away from the center. A partition of the confidence levels will be at the points where larger frequency changes occur. 


\subsection{Oblique views of transverse definition}

The oblique views are to test whether our designed oblique method shows any additional information which could help to verify the prostate definitions on the transverse slices. We expect that the oblique slices might better reveal the surrounding structures of the prostate in the oblique directions. We will compute two sets of the oblique slices, with one set cutting through the CT volume obliquely with a 45 degree angle against transverse parallel planes, and a -45 degree angle for the other set. Trilinear interpolation and rotational transformation methods are used in the computation of oblique slices and oblique contour definitions which will be presented in Section 3.6. The oblique technique has also inspired us to build a 3D contouring system to assist the physicians in their target definitions on the CT images. The contouring system will be presented in Chapter 4.

\subsection{Contouring system}

One of the issues that physicians face during contouring is that tissues may hide the organ. The goal is to design and implement a system that assists the physicians in contouring the target/organ. We propose a new concept of $3 \mathrm{D}$ contouring. The main idea is to compute different sets of oblique slices through the CT volume and enables users to draw contours of the target on both the transverse and oblique slices. Each contour is a sequence of points. The points are collected from all of the slices to form $3 \mathrm{D}$ point clouds which define the target. Then we can slice the point clouds to get combined definitions which can be super-imposed on the original CT slices. 
The combined definitions mean that the definitions are from the different views. The point clouds can be modified (by removing or adding points) as needed when editing is performed on the combined definitions.

The following subsections include the techniques on the oblique CT computation and the definition presentation on other views.

\subsubsection{Oblique CT computation}

The task is to compute a set of parallel oblique slices, given a set of the conventional parallel transverse slices (parallel to xy-plane). The oblique slices cut the set through the transverse volume. The computed oblique slices are perpendicular to the xz-plane. The desired angle is with respect to the xy-plane, in this way we obtain the set of the oblique slices intended. The resolution of oblique slices is the same as that in transverse slices, since physicians prefer to work on images with the same resolution without losing any anatomical information. The coordinate system of the patient is shown in Figure 3.8.

\section{Oblique by transformation}

The construction of an oblique slice depends on the angle that it forms against the transverse plane. The angle defines the desired views in order to improve the definition of the anatomical structures surrounding the target/prostate understudy. Therefore we need to compute the intensity values of the grid points on each oblique slice. Each grid point on the oblique slice will be located somewhere in a cube in the volume formed by the transverse slices. In this context, we will use transverse volume and transverse slices interchangeably, as they mean the same thing to us. 


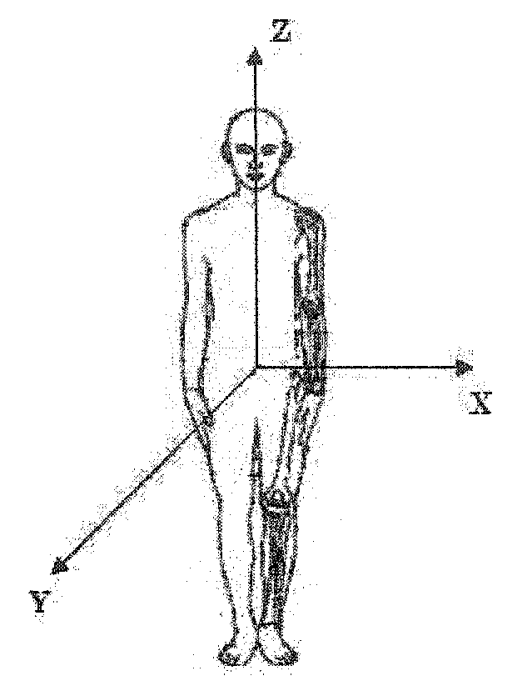

Figure 3.8: The coordinate system of the patient. (Courtesy of e-dummy/JIS)

We normally view a transverse slice in the direction perpendicular to the slice (e.g., into the paper). Thus we want to obtain a similar view for our oblique slices. In order to achieve it, we rotate the transverse volume by the angle mentioned above (e.g. $45^{\circ}$ ) through a rotation transformation $T$, such that the oblique slice will be in the $x y$-plane direction and we can now view it into the paper, just as we view the transverse slices. Figure 3.9 is a top view of our target volume and viewing direction is perpendicular to the $x y$-plane. The figure is a $2 \mathrm{D}$ illustration of a rotation by $45^{\circ}$ in order to provide a physician a simple and consisting way of looking at the slices [37].

The coordinates of a grid point on the newly rotated oblique slices correspond to that of a point in the original transverse volume through the rotational transformation $T$. Let $P$ be a point in the original volume, and be transformed to $P^{\prime}$ which happens to be a grid point in the transformed volume; then we can have: $T(P)=P^{\prime}$. Given $P^{\prime}$ 


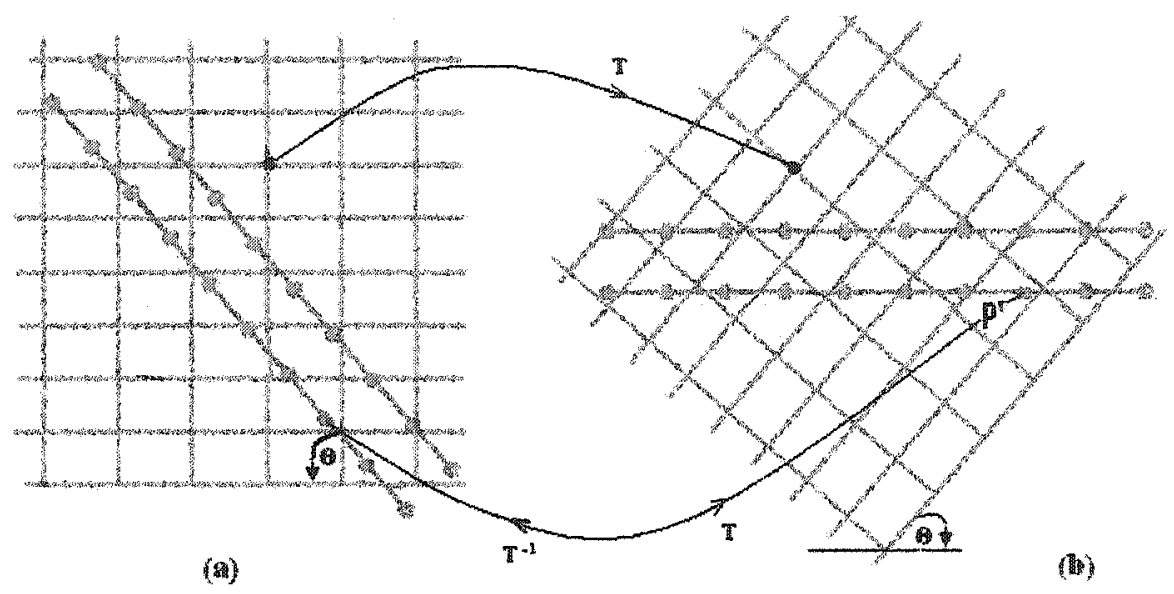

Figure 3.9: The oblique slice computation by transformation. The horizontal lines in (a) represent the volume slices, the two oblique lines represent the two oblique slices cutting through the volume. (b) is the result of the rotation of (a) by $45^{\circ}$. We rotate the grid by $45^{\circ}$ and then interpolate the original data to obtain the oblique slices.

(which is a grid point), we can obtain the coordinate of $P$, by applying the inverse transformation $T^{-1}$. That is $P=T^{-1}\left(P^{\prime}\right)$. Thus our problem for finding the intensity value of a grid point $P^{\prime}$ on the oblique slice can be re-stated as: a) obtain the inverse rotation transformation $T^{-1}$; b) compute the coordinates of $P$ in the original volume, by the formula $P=T^{-1}\left(P^{\prime}\right)$; c) compute the intensity value at $P$ by the trilinear interpolation, since $\mathrm{P}$ will not necessarily be a grid point in the transverse volume. The following is the algorithm for computing the intensities of the oblique slice [37]. 


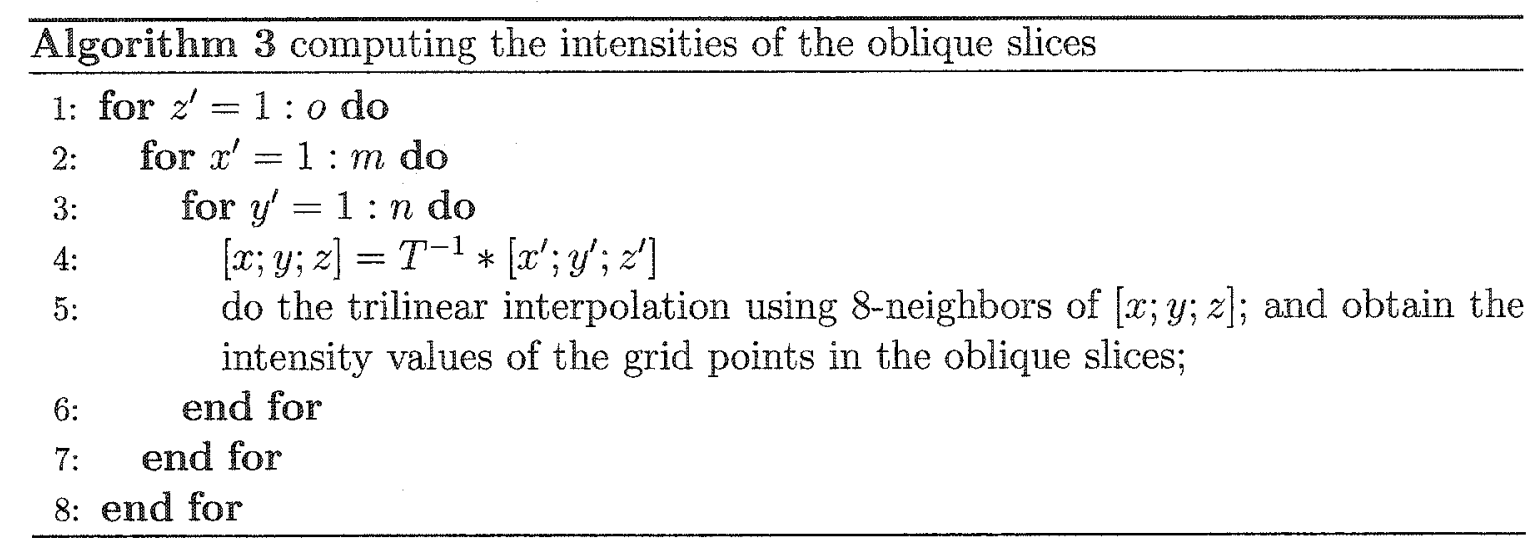

where $\left[x^{\prime}, y^{\prime}, z^{\prime}\right]$ are the grid coordinates in the oblique slices, and $[x, y, z]$ are the mapping of $\left[x^{\prime}, y^{\prime}, z^{\prime}\right]$ in the original volume, by applying the inverse transformation. $m, n, o$ are the sizes of the volume in each of the $X^{\prime}, Y^{\prime}, Z^{\prime}$ directions. Note that the multiplication in line 4 of Algorithm 3 can be turned into an addition or a substraction operation. Because the grid displacements in each of $x, y, z$ directions are the same. Thus only a couple of the grid coordinates need to be calculated by the multiplication of the transformation $T$ outside the three for loop in order to calculate $x, y, z$ displacements. The calculation of the remaining grid coordinates are done through the addition or substraction of the corresponding displacements. In this way, computation time is saved.

\section{Rotational transformation}

Our rotation of the oblique slices is around the $\mathrm{Y}$-axis, and the transformation can be expressed as the homogeneous transform $[38,39]$ of a $4 \times 4$ matrix. The top left $3 \times 3$ elements of the matrix corresponds to a rotation around $Y$-axis, as shown in the formula below: 


$$
T_{R}=\left[\begin{array}{cccc}
\cos (\theta) & 0 & -\sin (\theta) & 0 \\
0 & 1 & 0 & 0 \\
\sin (\theta) & 0 & \cos (\theta) & 0 \\
0 & 0 & 0 & 1
\end{array}\right]
$$

Angle of rotation is denated by $\theta$ (Figure 3.9). The inverse of the rotation transformation is given by the inverse of the matrix $T_{R}$, denoted as $T_{R}^{-1} \cdot T_{R}$ is an orthogonal matrix and therefore $T_{R}^{-1}=T_{R}^{T}$, where $T_{R}^{T}$ is the transpose of $T_{R}$.

\section{Trilinear interpolation}

The points in our CT volume lie on the cubic grids and the grid lines are parallel to the $\mathrm{X}, \mathrm{Y}$ and $\mathrm{Z}$ axes which form the cubic grid cells.

There are various interpolation methods available in the literature, nearest neighbor, trilinear and spline interpolations. The nearest neighbor interpolation is simple and fast, but is discontinuous. The spline interpolation technique performs very well when interpolating a value, but it is very time consuming, because it usually uses some higher order (e.g., cubic) polynomials to compute the coefficients.

A simple interpolation technique is the trilinear interpolation technique, which has continuity across the grids as well as good interpolation results. It is an extension of the 1D linear interpolation. Each pixel in a slice is represented by a point $p$ at the center of the pixel. When all slices are assembled together, they form a $3 \mathrm{D}$ volume where a cube is named voxel. Then $p$ becomes the center of the corresponding voxel. Figure 3.10 shows a pixel in the slice $S_{i}$ and the corresponding voxel in a volume. The trilinear interpolation process is as follows: firstly, eight points representing eight voxels are found such that the point $p$ is contained in the cubic formed by the 
points $q_{1}, \ldots, q_{8}$ (see Figure 3.11); secondly, four 1D line interpolations are performed between $q_{1}$ and $q_{2}, q_{3}$ and $q_{4}, q_{5}$ and $q_{6}$ and $q_{7}$ and $q_{8}$ to obtain the values for $q_{1}^{\prime}, q_{2}^{\prime}$, $q_{3}^{\prime}$ and $q_{4}^{\prime}$; in the third phase, two 1D interpolations are performed between $q_{1}^{\prime}$ and $q_{2}^{\prime}$ and $q_{3}^{\prime}$ and $q_{4}^{\prime}$ to obtain the values for $q_{1}^{\prime \prime}$ and $q_{2}^{\prime \prime}$ respectively; lastly the value of $p$ is found by the interpolation between $q_{1}^{\prime \prime}$ and $q_{2}^{\prime \prime}$.

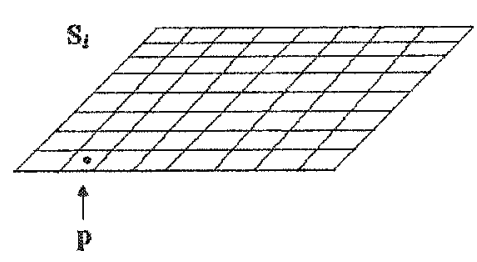

(a)

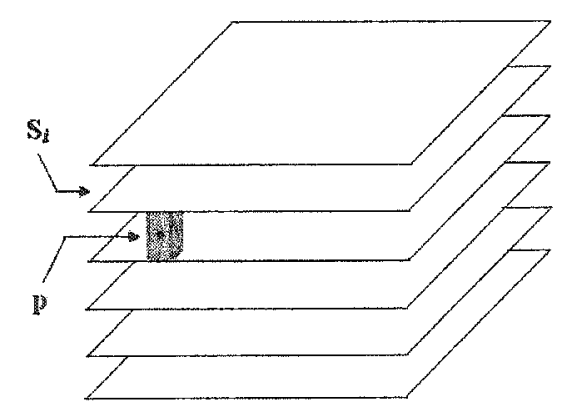

(i)

Figure 3.10: (a) a pixel with the center point $p$ in a slice; (b) the corresponding voxel with the center point $p$ in a volume.

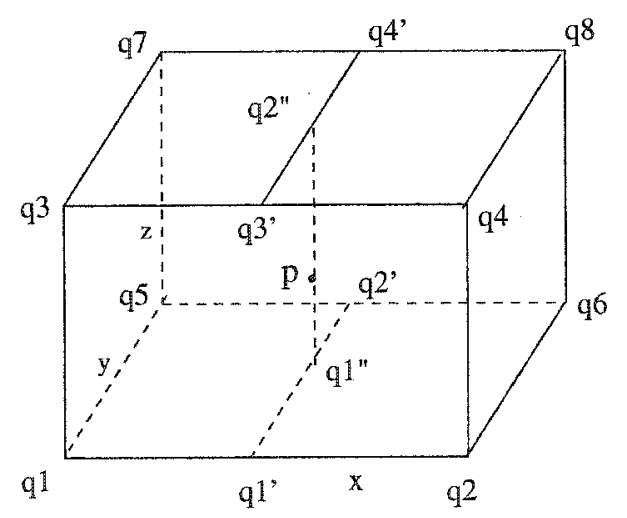

Figure 3.11: The figure above is the graphic illustration of the 3D trilinear interpolation of the point intensity 


\subsubsection{Organ/target Contouring Process}

The objective of the $3 \mathrm{D}$ contouring system is to enable the user (e.g., an oncologist) to accurately define and mark the organ for future uses (e.g., radiation treatment planning). In order to achieve this objective, we designed a contouring system which is based on simultaneous three-contouring-window system. One window displays the transverse slice and the other two windows display the oblique views (Figure 3.12). The main goal of the three-contouring-windows is to enable users to manually draw and edit contour lines on each window. The buttons (to foot and to head) are designed on each window to enable users to scroll through CT slices. Users choose one window to do contouring. Then the chosen window is defined as the primary window with the function buttons (e.g., contouring, clear contour).

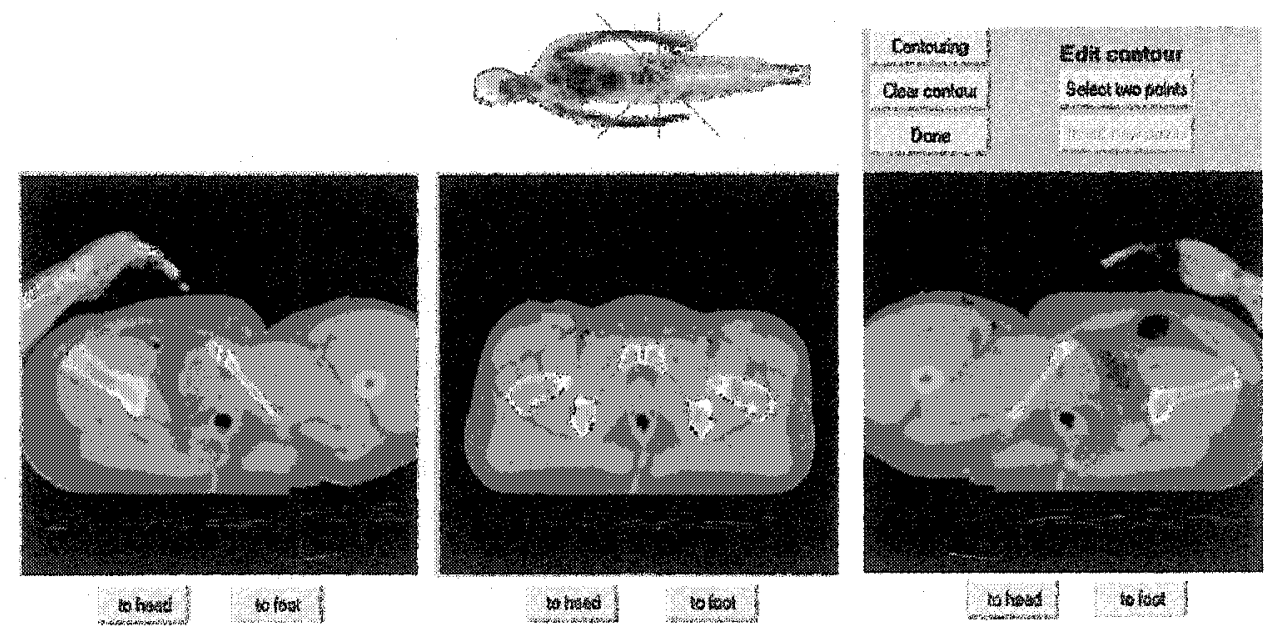

Figure 3.12: The snap shot of three contouring window system 


\section{Functionalities of contouring system}

\section{- Create a new contour}

The user starts the contouring process by clicking the contouring button on the primary window. The connected lines will be shown instantly as a user clicks and drags the mouse along the window. The contour will be closed as the user hits the ENTER key and finishes the drawing (Figure 3.13).

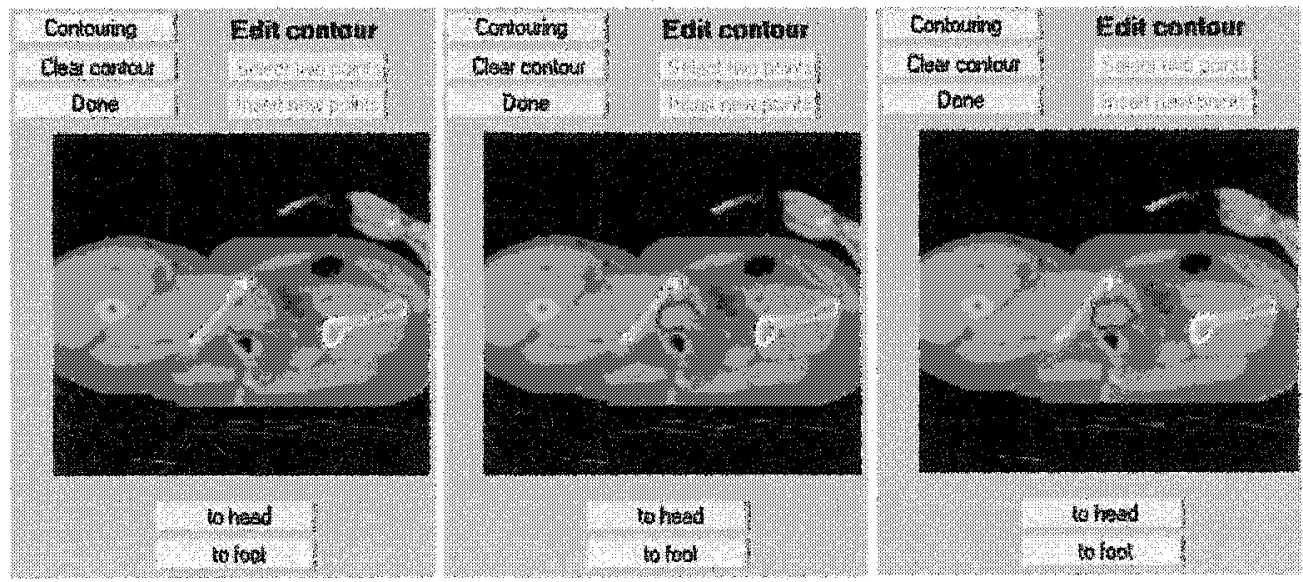

Figure 3.13: The process of creating ह new contour.

\section{- Clear contover}

The user clicks the clear contour button to delete the drawn contour. 


\section{- Edit contour}

\section{- Select 2 points}

The user can click the select 2 points button to enter into the contour editing mode and then click on the contour two times to select two points $(P 1, P 2)$. The shorter segment between the two selected points will be removed (Figure 3.14).

\section{- Insert new points}

The user is then required to insert some new points between the two selected points by clicking the insert new points button. The newly inserted points are considered to better define the target. The user hits ENTER key to finish inserting new points (Figure 3.14).

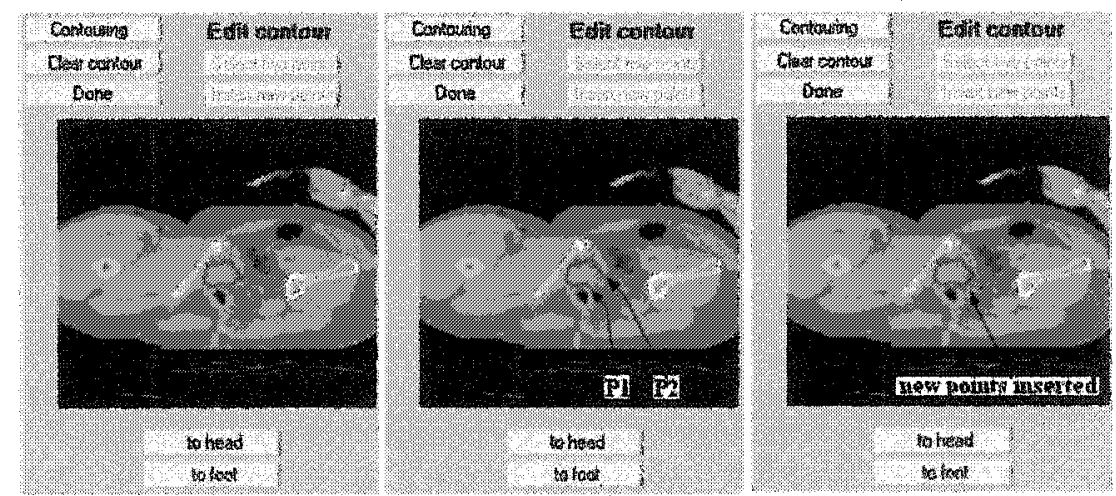

Figure 3.14: The process of editing a contour.

\section{- Terminate}

The user clicks the done button to save the drawn contour and terminate the contouring process. Clicking the done button also triggers to present the drawn contour 
on the other two windows which will be described in Section 3.6 .3 below.

\subsubsection{Presenting the definition on the other views}

In order to examine the correctness of the current target contour definition, we present the contour definition to the other viewing windows. If the contour definition is on the transverse slice, it is presented on the two oblique views; if the Contour Definition is on one of the oblique views, it is then presented on the other oblique view and the transverse view. What we see on the other views are some narrow segments which are the intersection of the current Contour Definition with the other viewing slices. The lengths of the segments depend on the slice thickness. Figure 3.15 gives a top view of the intersections of the transverse slices and the oblique slices, and the intersecting segments that we intend to compute. In this study, we choose the right oblique window as the primary window and define the target on the right oblique slices. When a contour drawing is done, we want to present the contour on the transverse window and the left oblique window. To achieve this, we compute the intersections between the current Contour Definition and the transverse slice, the current contour definition and the left oblique slice respectively. Here below we only describe the computation of the first pair of the intersection, as that of the second pair of the intersection is the same. 


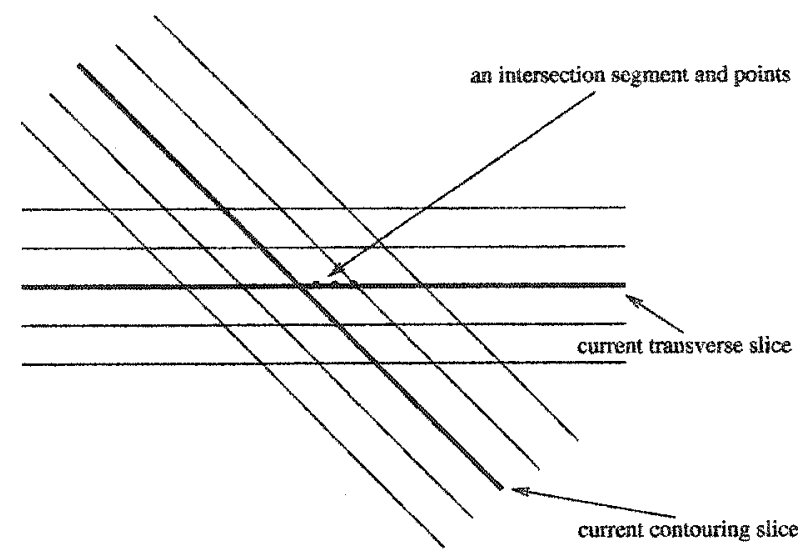

Figure 3.15: A top view: the oblique slices $\left(-45^{0}\right)$ intersects with the slices of the transverse.

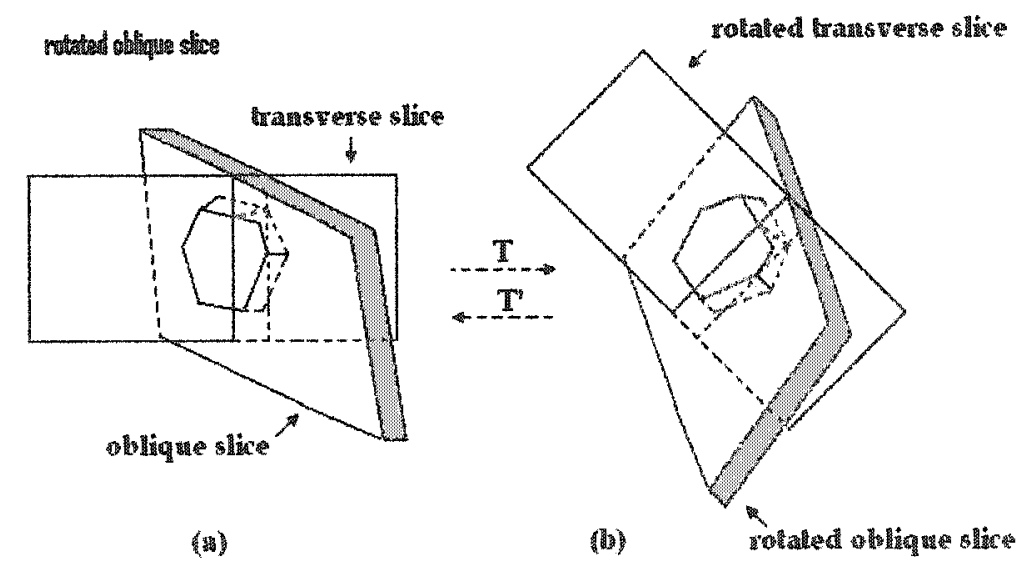

Figure 3.16: (a) The current transverse slice intersects the target contour on the current oblique slice $\left(-45^{\circ}\right)$ with the intersection segment in the red thick dots; (b) a rotation of $-45^{\circ}$ of (a) by the transfomation matrix $T$; the coordinates of exch grid point in the rotated transverse slice in (b) can be mapped into the coordinates in the original transverse slice in (a), using the inverse transformation T". 
Compute the intersection points of the transverse and the oblique slices

When the Done button on the drawing window is pressed, the area enclosed by the definition contour of the current oblique slice will be filled with the value 1 for the computation purpose. The current oblique slice has a thickness of $3 \mathrm{~mm}$, and it is intersected by a set of the transverse slices. Figure 3.16 shows a top view of the intersection of the current transverse and current defined contour. We need to compute the segment which is the intersection of the current slice with the definition contour on the current oblique slice. The segment's length is about $3 \mathrm{~mm}$, since it is contained within the slice thickness.

The area of a prostate contour is relatively small and occupies a small percentage of a slice. Thus we can consider to include only the relevant region (region of interest) in our segment computation to increase the speed. Let the region of interest (ROI) on the current transverse slice be $A$, imagine that we rotate $A$ with the rotational $\left(-45^{\circ}\right)$ transformation $T$ (described in the Section 3.6.1), and get a transformed ROI named $A^{\prime}$, on a new slice. 


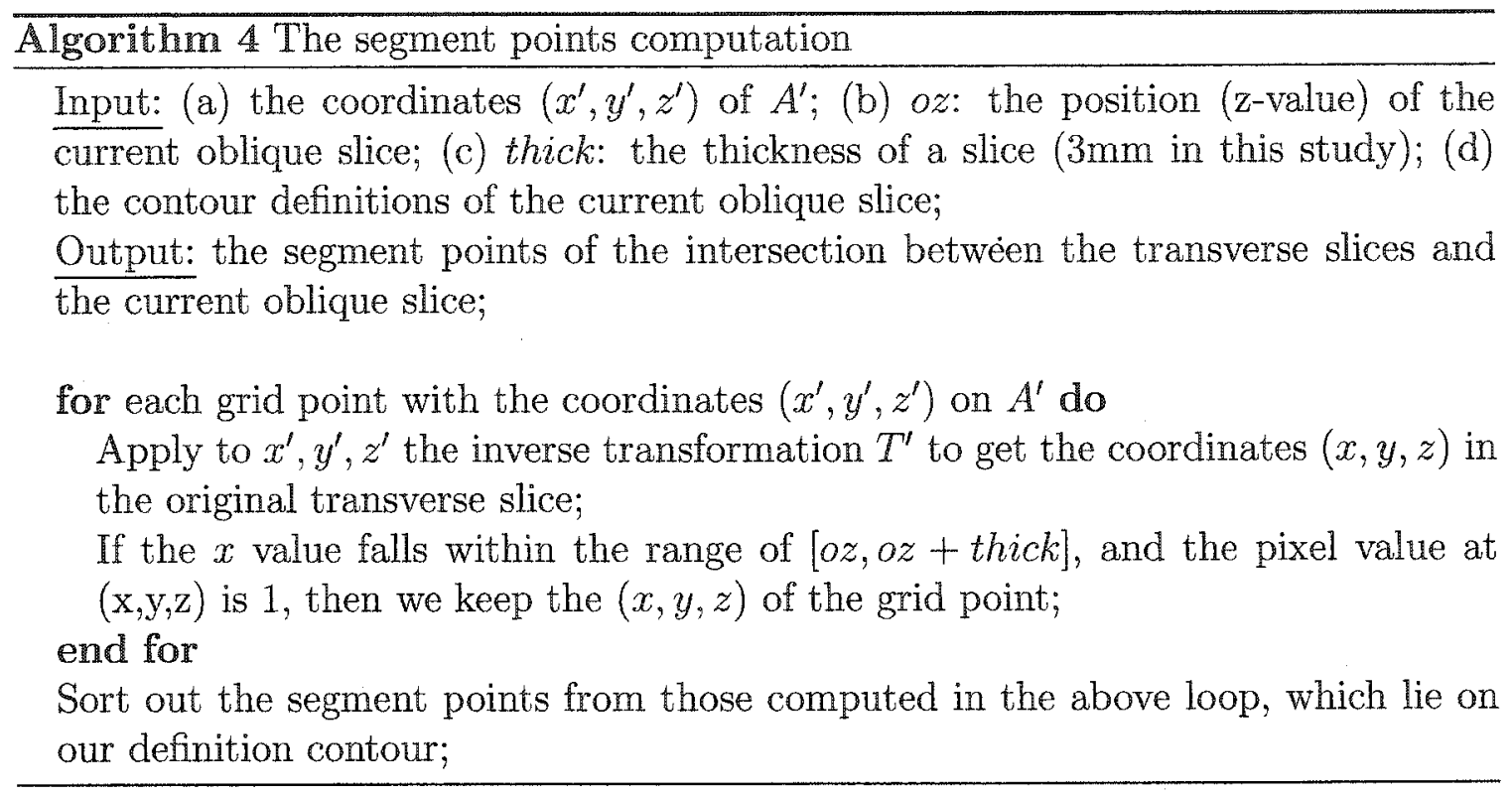

Time complexity of Algorithm 4: $\Theta(m n)$, where $m, n$ are the number of rows and columns in our ROI, which is relatively small.

This chapter presented our work on the evaluation system and the 3D contouring system. The evaluation system includes 3D surface reconstruction, volume computation, 3D image registration and evaluation statistics for the physicians' prostate definitions and the oblique presentation of the transverse target definition. The contouring system includes oblique CT computation, target contouring process and presentation of the definitions on the other viewing windows. The systems development is presented in Chapter 4. 


\section{Chapter 4}

\section{System development}

This chapter describes the development of the two tools that we have designed: the evaluation tool and the contouring tool. The physician evaluation tool is a tool for assisting the physicians' target and organ definitions in 2D and 3D for the error analysis. Our contouring tool uses a novel idea for which enables the physicians to contour in 3D instead of 2D. We will describe each tool individually. Each tool's descriptions include an overview of the tool, tool components and component designs. We also discuss the systems' environment setup and some issues of the systems.

\subsection{Evaluation system/tool}

The evaluation tool aims at presenting the physicians' prostate definitions in $2 \mathrm{D}$ and $3 \mathrm{D}$, and providing the error estimates visually and statistically. The following sections present the system overview and the system component designs with the design flow 
diagrams.

\subsubsection{Overview of physician evaluation system/tool}

This tool enables research to assess the quality of the target/organ contours by the physicians. The evaluation tool consists of eight components: 3D surface reconstruction, $3 \mathrm{D}$ rigid registration, volume and statistics computation, computation of the oblique CT slices, computation of the oblique definitions, $2 \mathrm{D} / 3 \mathrm{D}$ visualization, and oblique presentation. The CT images, the Photo images, their definitions and the oblique angle are the input of the system. The visual images and the statistics are the output. Figure 4.1 gives an overview of the system. Each of the components is described in the following subsections.

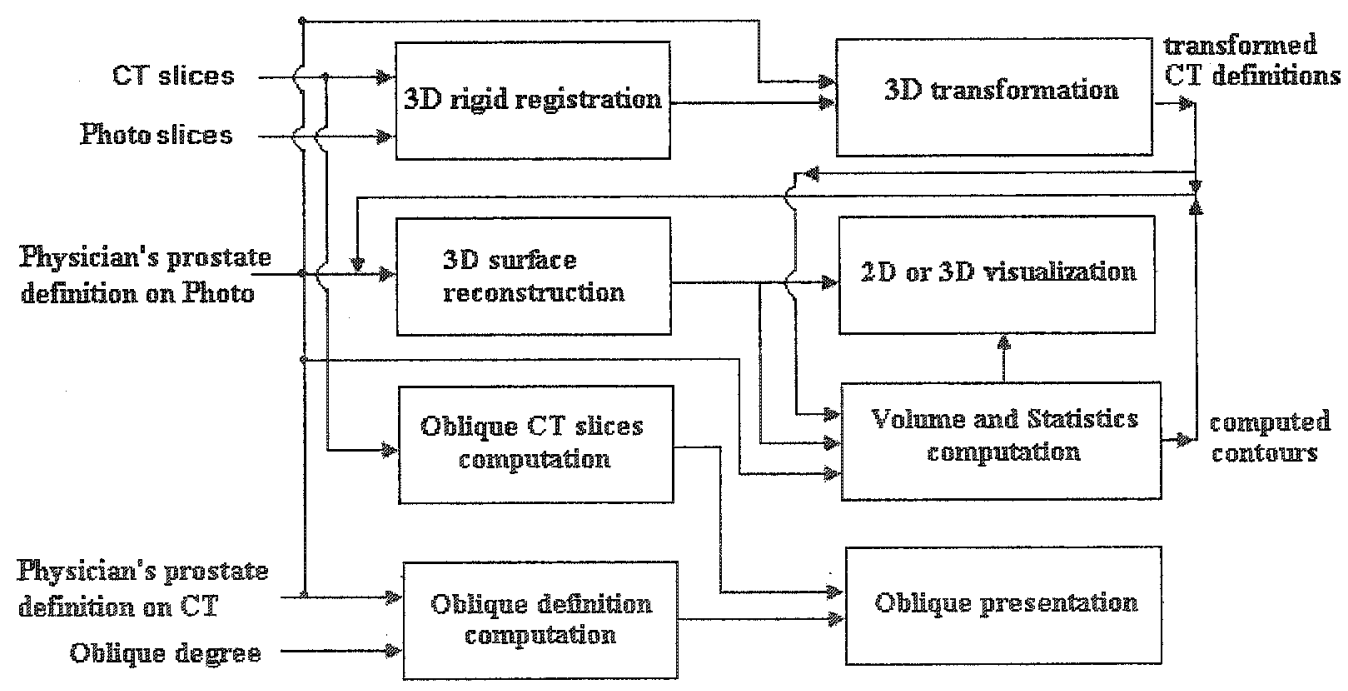

Figure 4.1: The evaluation system overview. 


\subsubsection{Component design}

This section presents the designs of the components in our evaluation system. Each component consists of several modules and each module has some specific functionalities. We will describe the functionalities of the modules in each component and their relationships with other modules.

\section{$3 D$ surface reconstruction $(3 D S R)$}

The $3 D S R$ component is used to create the tiles of triangles for the reconstruction of the 3D surface of the target. It consists of the two modules: Initial points computation (IPC) and Triangulation as shown in Figure 4.2. The IPC module computes the two initial points of the triangulation using the method described in Section 3.1. The Triangulation module implement the shortest diagonal algorithm [1]. The input for the 3DSR are the physicians' CT and Photo definitions (in the forms of contour points) of the target. The output are the point sets of the tiles of triangles described in Section 3.1.

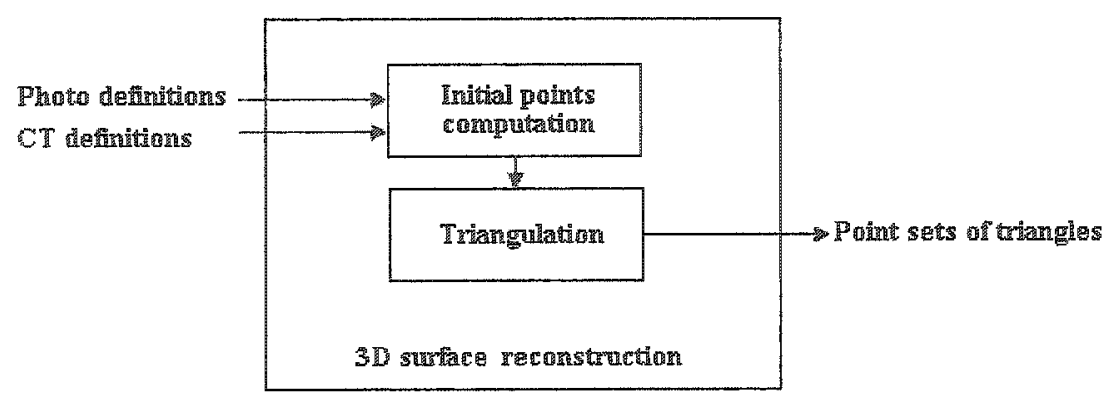

Figure 4.2: The 3D surface reconstruction (3DSR). 


\section{$3 D$ rigid registration $(3 D R)$}

The $3 D R$ component is used to register the two sets of different images. It implements Algorithm 2. The 3DR component consists of the four modules: Multi-resolution, rigidTransform, MIfunc and fminsearch. The Multi-resolution module re-scales the two sets of the input volumes with a resolution scale at the early stage of the registration. The rigidTransform module implements the six parameter homogeneous transformation and the partial volume trilinear interpolation methods described in Section 3.3 and it computes the joint histogram of the two modalities. The MIfunc implements the normalized mutual information [11], it calculates the mutual information value of the two modalities using the joint histogram. The fminsearch (implemented in Matlab's optimization toolbox) takes the mutual information value as its input parameter and compute iteratively a set of the estimation parameters. The program will stop when the computed estimates satisfy the accuracy threshold. Figure 4.3 gives the overview of the $3 D R$ component.

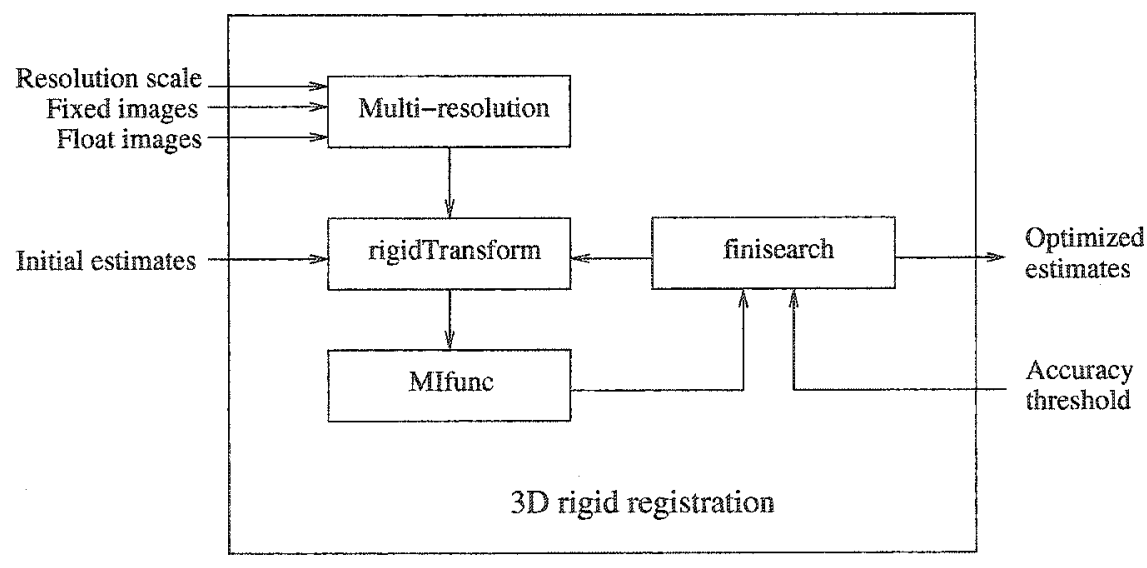

Figure 4.3: The 3D rigid registration $(3 D R)$. 


\section{The $3 D$ transformation $(3 D T)$}

The $3 D T$ component is used to transform an input volume into a new volume by applying a set of transformation parameters. It consists of the transform $3 d$ module. The module's input are the volume to be transformed and the optimized transformation parameters computed in the registration component just described.

\section{The volume and statistics computation (VSC)}

The VSC component is used to compute the definition volumes of the target and a set of the statistics for the evaluation of the physicians' target definitions. The VSC component consists of the two modules: getVolume and getStats. The getVolume module implements the volume computation Algorithm 1 which is described in Section 3.2 and it computes the volumes between the pairs of the adjacent slices. The input of the module are the tiles of the triangles computed from the $3 D S R$ component. The getStats module takes the Photo definitions, the transformed CT definitions and the calculated volumes as its input parameters, and computes the statistics. The module implements the ray-shooting method to compute the 3MSTD of the definition contours. The minimum, maximum error gaps between the two volumes, and their error directions are also computed. The 3MSTD definitions computed are then used to form the 3MSTD definition volumes for the visual presentation of the error estimates. Figure 4.4 gives the overview of the VSC component.

\section{The $2 \mathrm{D}$ or $3 \mathrm{D}$ visualization $(2 D 3 D V)$}

The $2 D 3 D V$ component is used to present the visual images of the target definitions 


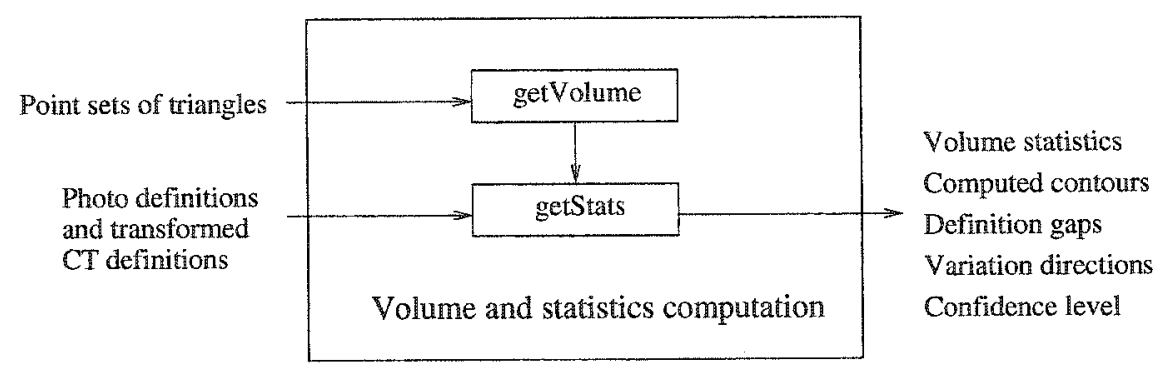

Figure 4.4: The computation of the volume and statistics (VSC).

in 2D and 3D. It consists of the modules: 2 Ddraw and 3Ddraw. The 2Ddraw module presents the visual images of the target definitions in 2D. The module's input are the definitions includes the 3MSTD definitions. All of the definitions for a target slice can be drawn and overlayed together for the visual variation analysis. The 3Ddraw module presents the 3D reconstructed target surface. The module's input are the tiles of the triangles from the $3 D S R$ component. The two registered volumes can be drawn together using different color schemes for each volume. We use Matlab's holdon function to perform the overlayed drawing. The holdon function is to inform the window system not to overwrite a previous drawing while a new drawing is performed. Figure 4.5 gives the overview of the $2 D 3 D V$ component. 


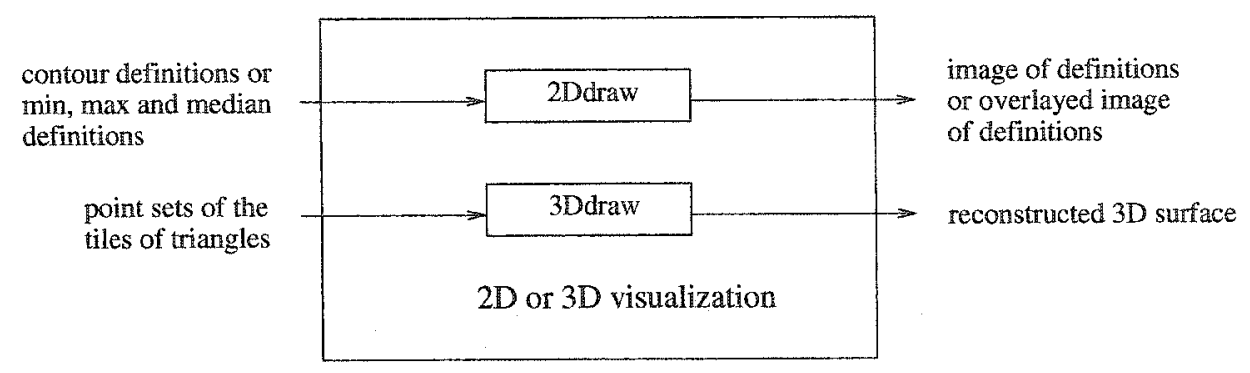

Figure 4.5: The 2D or 3D visualization (2D3DV).

\section{The oblique CT computation (OCTC)}

The OCTC component implements Algorithm 3 and it computes the oblique CT slices with an angle to the transverse slices. The component consists of the modules: InvT and rot512BySplit. The InvT module is responsible for obtaining the inverse rotational transformation matrix with the oblique angle from the input. The rot512BySplit module implements the trilinear interpolation method to compute the grid point intensity values of the oblique slices. The module's input are the anatomical CT slices and the inverse matrix from the InvT module. The module's output are the oblique CT slices with the desired oblique angle. The module also uses divideand-conquer method to solve the insufficient memory problem. Each time only part of the 512 slices are loaded into the memory to compute a set of partial oblique slices, and several sets of the partial slices make a full set of the oblique slices. Figure 4.7 gives the overview of the OCTC component.

The oblique definition computation $(O D C)$

The $O D C$ component computes the definition points of the intersections between the current transverse definition and a set of oblique slices. The component consists 


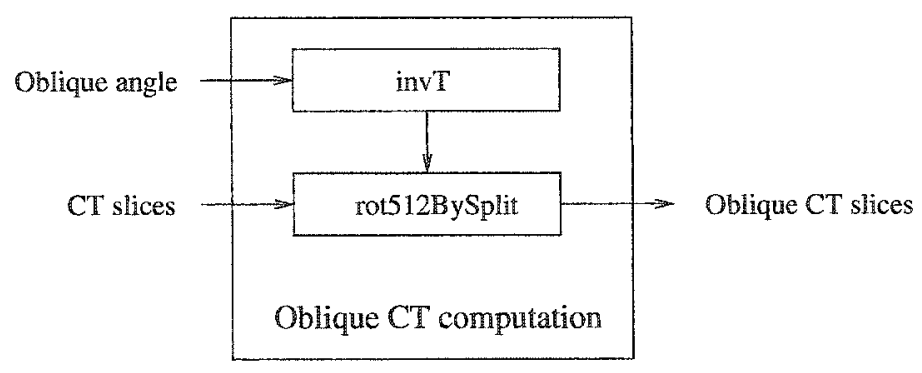

Figure 4.6: The oblique CT computation (OCTC).

of the modules: InvT and doTransDef 4 obliq. The InvT module is responsible for obtaining the inverse rotational transformation matrix with the oblique angle from the input. The doTransDef4obliq module implements the method for computing the intersection points using the inverse matrix obtained from the InvT module. The method is described in Section 3.6.3. Figure 4.7 gives the overview of the $O D C$ component.

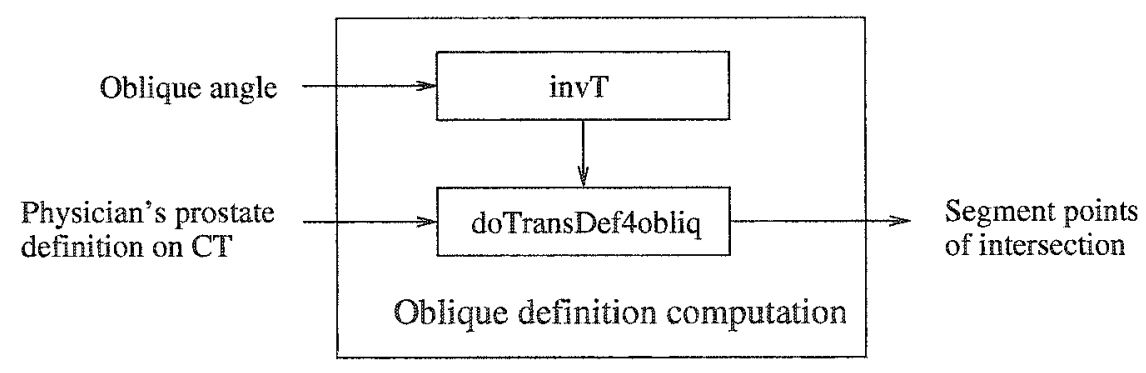

Figure 4.7: The oblique definition computation $(O D C)$.

\section{The oblique presentation of definitions $(O P D)$}

The $O P D$ component presents the oblique images of the transverse slices, with the definition intersection points superimposed. The input of the OPD component are the transverse CT slices, the transverse CT definitions, the oblique CT slices from 
the $O C T C$ component, the definition intersection points from the $O D C$ component. The oblique images are displayed alongside with the transverse definition images. The $O P D$ component consists of the module of disp3plotIn 3 and three window objects: $C f, L f, R f$. The disp3plotIn 3 module creates the three window objects and stores the input and intermediate data in these objects. The module also creates to foot and to head buttons on each of the object windows. Each object has a set of the interfaces such that the user can traverse the target slices by clicking the buttons (to foot and to head). The disp3plotIn3 responds to the button(to foot or to head) click and activates the corresponding interface of the object. The objects can also communicate with one another. Each object keeps track of the references of the other objects and the index of the currently displayed slice, and have the responsibility of refreshing and updating the other two window images when the status of its own gets changed. Figure 4.8 gives the overview of the $O P D$ component.

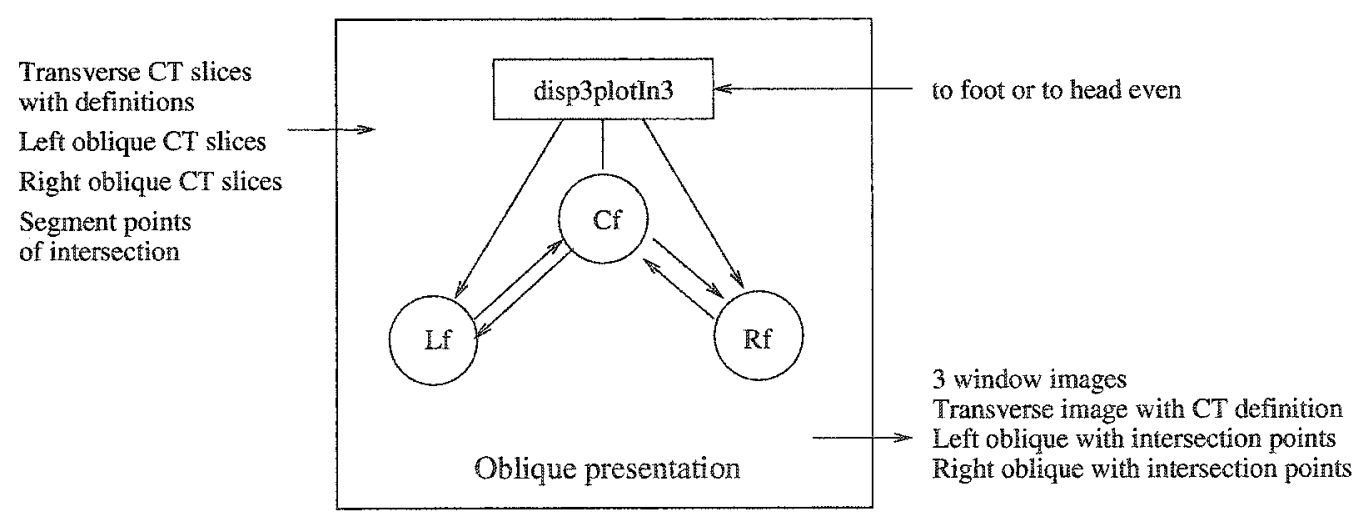

Figure 4.8: The oblique presentation of definitions (OPD). 


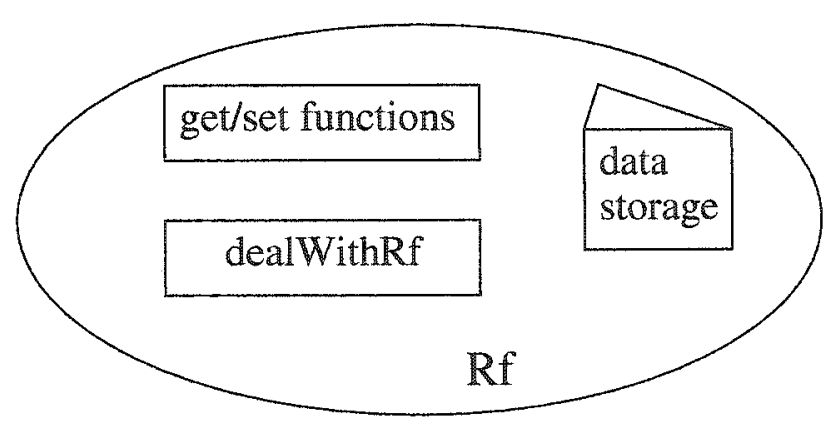

Figure 4.9: The $R f$ object and its interfaces.

Figure 4.9 gives the overview of the $R f$ object in the $O P D$ component. The $R f$ window object consists of the module of dealWith $R f$ for the updating and refreshing the window images and their target definitions when activated by the disp3plot In 3 module which responds to an external user input (to foot and to head). The datastorage is used to store the user-data such as the slice index and the object references. The get and set functions are Matlab functions to get and set the object data.

\subsection{Contouring system}

The system has the capabilities of the manual contouring, contour editing, and presenting of the effect of the current slice definition on the other views (the transverse or the oblique). 


\subsubsection{System overview}

The contouring system consists of four components: Oblique CT computation, manual contouring, definition presentations on other views and visualization. The CT images, and the oblique angle are inputs to the system. The system is designed to use three windows to display the transverse and the oblique images. The CT prostate definitions are super-imposed on their corresponding CT images. Figure 4.10 gives an overview of the system.

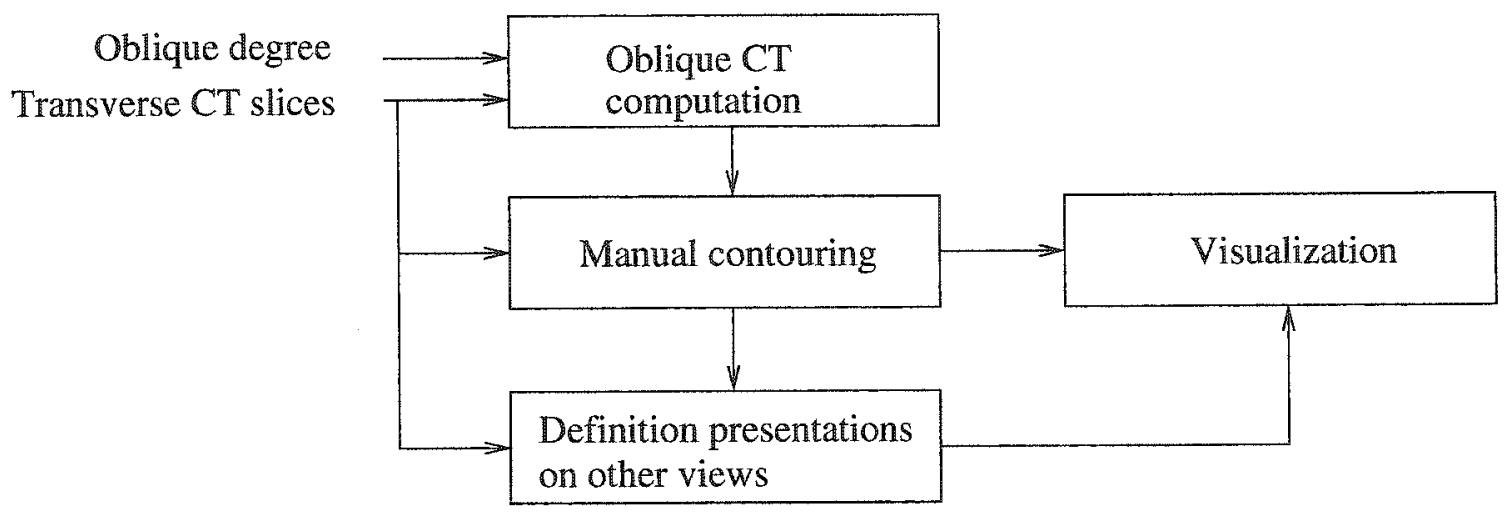

Figure 4.10: The contouring system overview.

\subsubsection{Component design}

This section presents the designs of the components in our contouring system. Each component consists of several modules and each module has some specific functionalities. The descriptions of the components and their modules are given respectively. 


\section{The oblique CT computation $(O C T C)$}

The OCTC component computes the oblique CT slice with an angle to the transverse slices. The component design is the same as that in Section 4.1.2.

\section{The Manual contouring on right oblique images(MCROI)}

The MCROI component is used to manually draw contours on the right oblique window. The input of the component are the transverse $\mathrm{CT}$ slices, and the oblique CT slices from the OCTC component. The MCROI component consists of the module of disp3In3ro and three window objects: $L f, C f, R f$. The disp3In3ro module creates the three window objects with three windows located on the left, the center and the right of the screen respectively. Each object window has the to foot and to head buttons. The three object windows display the left oblique images, the transverse images and the right oblique images respectively. The to foot and to head buttons on each of the object window are used for traversing through the CT slices. In this thesis, we describe the contouring on the right oblique window. The disp3In3ro module also creates some specific buttons for the manual contouring (MC) on the oblique drawing window. The MC buttons are contouring, clear contour, select 2 points, insert new points, and done. Figure 4.11 gives the overview of the $M C R O I$ component, and Figure 4.12 gives the overview of the $R f$ object. The $R f$ object has a set of interface functions which corresponds to the MC buttons. The functions are contouring, clearcontour, select2points, insertnewpoints and done. These functions are activated by the disp3In3ro module in response to the button clicks by the user on the oblique window. The contouring function is responsible for the contour drawing process. The user hit ENTER to stop the process and the drawn 
contour is stored in the user datastorage area. The clearcontour function clears the drawn contour for redrawing. The functions of select2points and insertnewpoints are for the contour editing purpose. The select 2 points function selects two points and the shorter segment between these two points is removed. Then the insertnewpoints is used to add new points to replace the removed segment. The done button is used to finish the contouring and also initiate the presentation of the drawn contour on the other two viewing windows. The presentation will be described below.

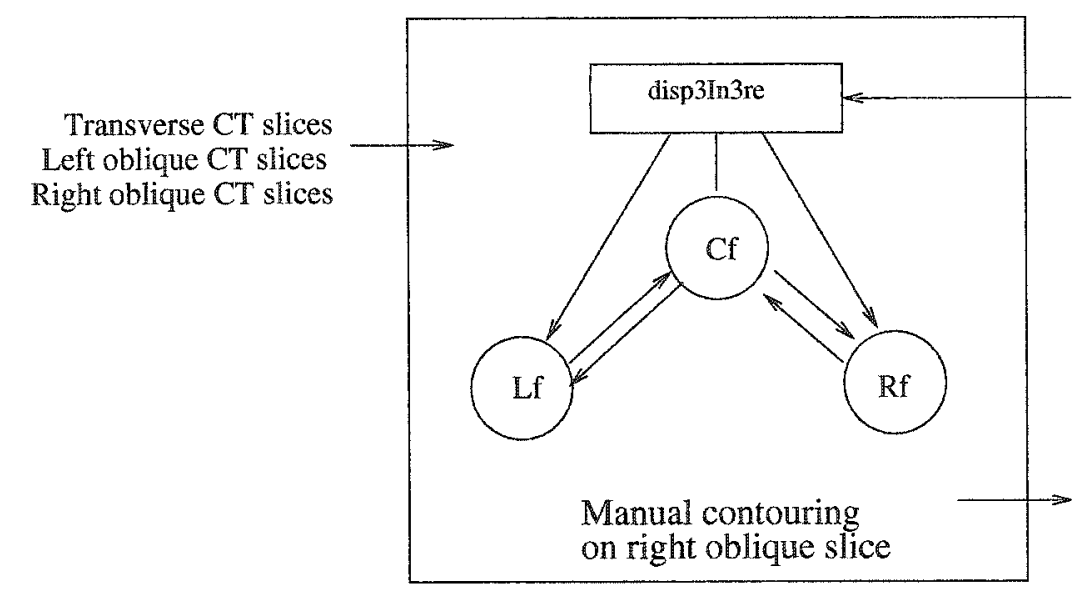

The events: to foot or to head contouring clear contour select 2 points insert new points done

3 window images:

Transverse with intersection points Left oblique with intersection points Right oblique with CT definition

Figure 4.11: The manual contouring on the right oblique images.

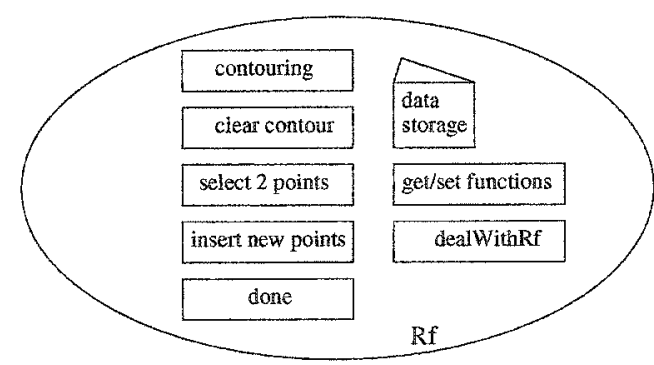

Figure 4.12: The $R f$ object and its interfaces for the manual contouring and the definition presentation on the other views. 


\section{The Presentation of the definitions on other views(PDOV)}

The PDOV component is used to compute the intersection points between the current definition with the viewing slices on the other viewing windows. The PDOV component is activated by the MCROI component with the click of the done button on the oblique window. The PDOV component consists of the module: invT, computeTCRO4dcpnts and computeTCRO4dcpnts. The InvT module is responsible for obtaining the inverse rotational transformation matrix with the oblique angle as its input parameter. The computeTCRO4dcpnts and computeTCRO4dcpnts implement Algorithm 4. The input for computeTCRO4dcpnts module are the inverse matrix from the InvT module, the transverse CT slices and the current contour definition. The computeTCRO4dcpnts module computes the intersection points between the current definition oblique slice and the transverse CT slices. The input for computeLCRO4dcpnts module are the inverse matrix from the InvT module, the left oblique CT slices and the current contour definition. The computeLCRO4dcpnts module computes the intersection points between the current definition oblique slice and the left oblique slices. The intersection points are then presented in the corresponding images when the done button on the definition window is pressed. Figure 4.13 gives the overview of the done interface function of the $R f$ object.

\section{The visualization}

The visualization component presents the visual images of the target definitions in the three windows. Matlab's plot and holdon functions are used to overlay the definitions and computed intersection points on the images in the corresponding viewing windows. 


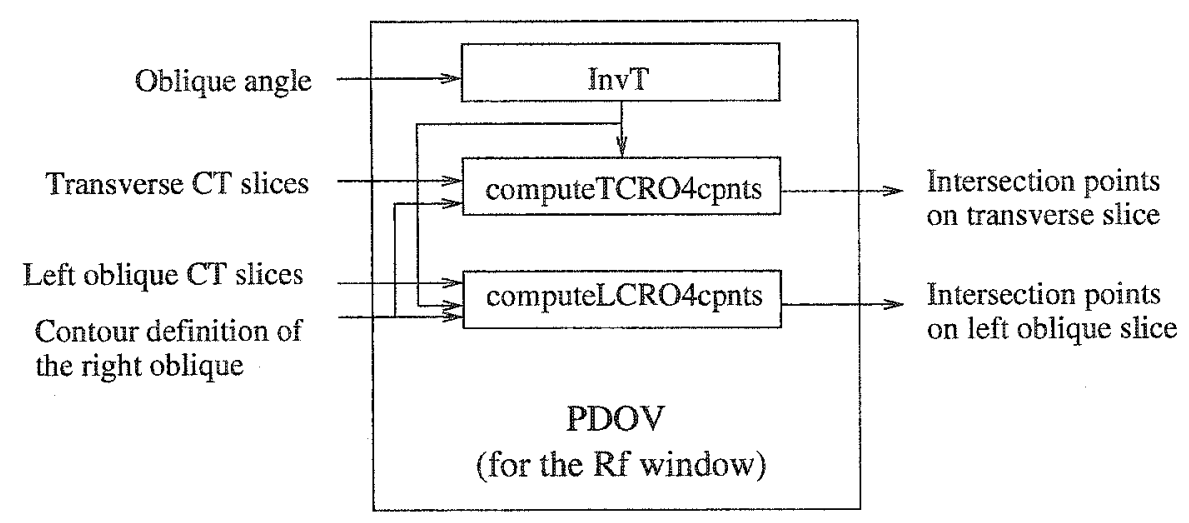

Figure 4.13: The PDOV component design diagram.

\subsection{Environment setup}

In the systems, data files, temporary files and program files are stored in different directories in a hierarchy order. Therefore the directory paths need to be setup properly at the start of the system. The program setallpath.m is designed to set up all the directories. The user only needs to change a prefix variable which stores some upper level directory names (e.g., c: \users). The examples are given in the program header.

There are some parameters and values which are used in the systems. To provide a common access point of the parameters and values, the getCommonPara.m routine is designed to set up the parameters and values for the system. Other programs can obtain the needed parameters and values by executing 'getCommonPara.m'. Some typical parameters and values are CT image resolution, slice thickness, center slice, physicians' names, number of definitions for each physician, etc. The advantages of that are: first users do not have to enter them at run time; second users do not have 
to declare any global variables in the system, thus the system resources are saved.

\subsection{Issues of the contouring system}

We are aiming to explore and compute the oblique views with the arbitrary angles in real time. So far we are pre-computing our $45^{\circ}$ and $-45^{\circ}$ oblique volumes, due to the considerable time requirement in computing the oblique volume. The $512 \mathrm{CT}$ transverse slices are needed to compute each of the 50 oblique slices (sizes of $512 \times 512$ ) and the computation takes about 10 minutes in our lab with $1 \mathrm{G}$ memory. Matlab plays a large role of the slow computation, because it tends to use a significant amount of memory and it is very slow in executing for-loops and while-loops. In order for the system to be real time and applicable in a clinical setting, we need first to reduce the computation time and memory usage. We will discuss this issue more after our experiments in Chapter 5.

This chapter presented our work on the error estimates, and our contouring system. The evaluation presents the error analysis in $2 \mathrm{D}$ and $3 \mathrm{D}$. The contouring tool includes the oblique volume computing, the manual contouring, the presentation of the definitions on the other views, our new idea of $3 \mathrm{D}$ contouring, and some issues of the system. 


\section{Chapter 5}

\section{Experiments}

In this chapter we present the experiments conducted on the system operation and the target definition evaluation of a prostate as well as the demonstration of the contour evaluation system. The experiment was conducted using Matlab and the experimental data are the CT, the Photo and the physicians' prostate definitions (courtesy of Jeff Gao, ORCC).

\subsection{Data source}

We are using the data from Visible Human Project [21] and Jeff Gao (a Carleton's Phd student who is currently preparing his thesis at the ORCC) for testing our evaluation tool and contouring tool. We have been granted the permission by the National Library of Medicine in the U.S. for the use of the Visible Human Project Data (male) in our research. The data used in our research includes the CT and the 
Photo images of the visible human male.

Gao collected a set of data in his evaluation study, in which an urologist, an oncologist and an anatomist were invited to define the prostate target on the anatomical Photo slices of the visible human male. The definitions are regarded as the gold standard and are regarded as the major difference from the other target delineation researches. The CT images of the visible human male were provided to three radiation oncologists and they were asked to contour the target following the ORCC's protocol. Each physician defined the prostate target twenty times. The definitions of prostate by the radiation oncologists are for the error evaluation of the physicians' target definitions.

Acquisition of transverse CT and Photo images has been completed using male cadavers. The male was sectioned at $3 \& 5$ millimeter intervals for CT and one millimeter intervals for Photo. The CT data consists of the axial CT scans of the entire visible human body taken at a resolution of $512 \times 512$ pixels, where each pixel is made up of 12 bits of grey tone. Whereas the Photo data consists of the axial anatomical images are $2048 \times 1216$ pixels where each pixel is defined by 24 bits of color, and each image has a size of 7.5 megabytes. The Photo anatomical cross-sections are at $1 \mathrm{~mm}$ intervals. There are 1871 cross-sections for both CT and Photo. The pixel sizes of CT and Photo images are 0.8984 and $0.33 \mathrm{~mm}$ respectively. The complete male data set, 15 gigabytes in size, was made available in November, 1994 [21]. About 200 CT and 500 Photo images, covering the entire prostate, are selected for this research work. 


\subsection{The evaluation system}

This section presents the experiments on our evaluation system, which include the system operation, the prostate surface reconstruction, the prostate volume computation, the $3 \mathrm{D}$ registration of the $\mathrm{CT}$ and the Photo images, the evaluation statistics and the oblique presentation.

\subsubsection{System operation}

Figure 5.1 gives the screen flow diagram to show the hierarchy flow control of the evaluation system. The description of the system operation is given below.

A user starts the systems by executing the main program EvalDemo.m in Matlab and the main menu (a) of Evaluation and Contouring appears (Figure 5.1). There are two system options on menu (a): Definition Evaluation and Prostate Contouring. The user clicks on the Definition Evaluation option to start the evaluation system and the 3D Evaluation menu (b) appears. Menu (b) has two operation options: Transverse Evaluation and Oblique Presentation. The first option is to evaluate the physician definitions which are conducted on the transverse slices. The second option is to present the transverse definitions on the oblique slices. When the user clicks on the first option on menu (b), the Transverse Evaluation menu $(d)$ appears. Menu $(d)$ has three operation options: $3 D$ Reconstruction, $3 D$ registration and Error Analysis. The first option on menu $d$ is to reconstruct the $3 \mathrm{D}$ surfaces of the target; the second option is to register the two volumes (e.g. CT and Photo) in 3D domain; and the third option is to compute a set of the statistics and images for the error 


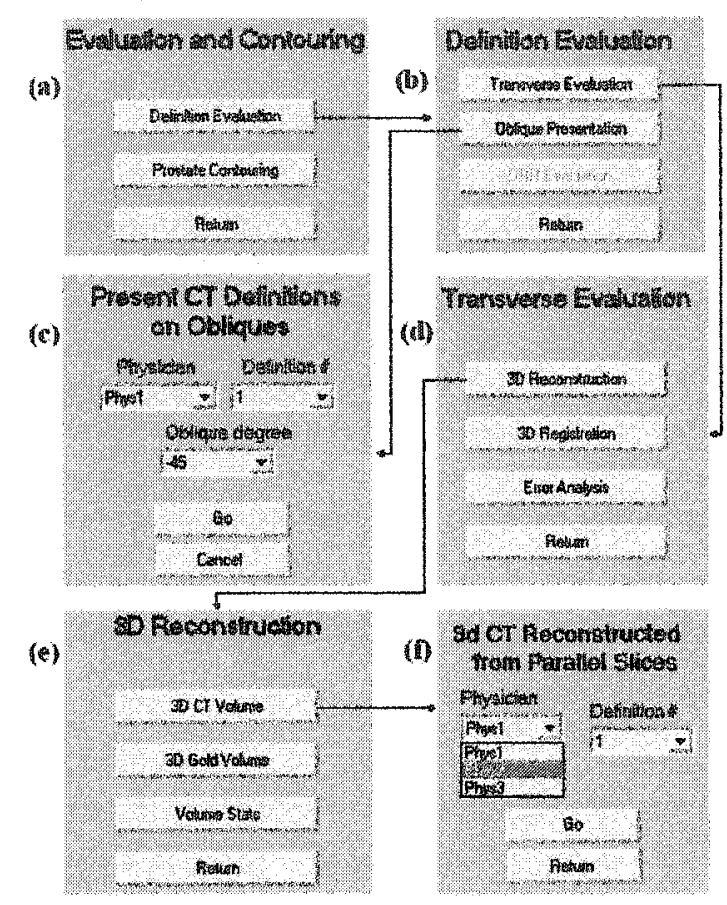

Figure 5.1: The control flow of the evaluation system. The dimmed button indicates that the module is included, but not yet implemented in this study.

analysis of the target definitions from the physicians. When the user clicks on the first option on menu (d), the $3 D$ Reconstruction menu (e) appears. Menu (e) has three operation options: $3 D$ CT Volume, 3D Gold and Volume Stats. The first option is to reconstruct the 3D surfaces of the CT definition volume; the second option is to reconstruct the $3 \mathrm{D}$ surfaces of the Photo definition volume; whereas the third option is to compute the 3MSTD of the CT volumes for the comparison between the volumes of $\mathrm{CT}$ and the volume of Photo. A parameter menu $(f)$ appears when the first option on menu (e) is clicked. With menu $(f)$, the user can choose one of the physicians who did the target definitions, and a definition sequence number. In this 
system, we use the definitions from three physicians, and each physician has twenty definitions. Once the parameters are chosen, the user can click the go button to start the $3 \mathrm{D}$ reconstruction and volume computation. When the user clicks on the second option on $(b)$, the Oblique Presentation menu $(c)$ appears. The Oblique Presentation option is to present a physicians transverse definitions on the oblique slices, such that some hidden structures may be revealed for examining the correctness of the transverse definitions. With menu $(c)$, the user can choose a physician and his definition sequence number. The user clicks the go button to start the presentation. For each menu mentioned above, there is a Return or Cancel button designed for the user to return back to the previous menu.

We present our experimental results of the evaluation system in the next subsections.

\subsubsection{Prostate reconstruction}

\section{D prostate of the CT and the Photo}

The 3D prostate surfaces of the CT and the Photo have been reconstructed from the parallel definition contours using the algorithm described in Chapter 3. The algorithm connects the created 3D triangles using the contours of the slices. The input to the prostate reconstruction module are the physicians' CT and the Photo definitions of a prostate. The definitions are contours which are in the form of points and stored in the text files. In the process of the reconstruction, the contour points of $\mathrm{CT}$ and Photo are loaded into the memory. The pair of the adjacent contours are centered and scaled to a unit square such that the resulting contours have a common center and a similar size. Then the initial two points of the triangulation are computed using 
the ray-shooting method described in Section 3.1. The reconstruction algorithm then is executed to create a tiles of triangles. At last, Matlab's plot3 procedure is called to draw the triangles and generate the $3 \mathrm{D}$ surface. The measure of the plot is in centimeters and the diameter of a contour in a slice is around $3-6 \mathrm{~cm}$. A viewer has the choice to select one of the sixty definitions from the three physicians for a 3D prostate display. A $3 \mathrm{D}$ plot is shown in figure 5.3:

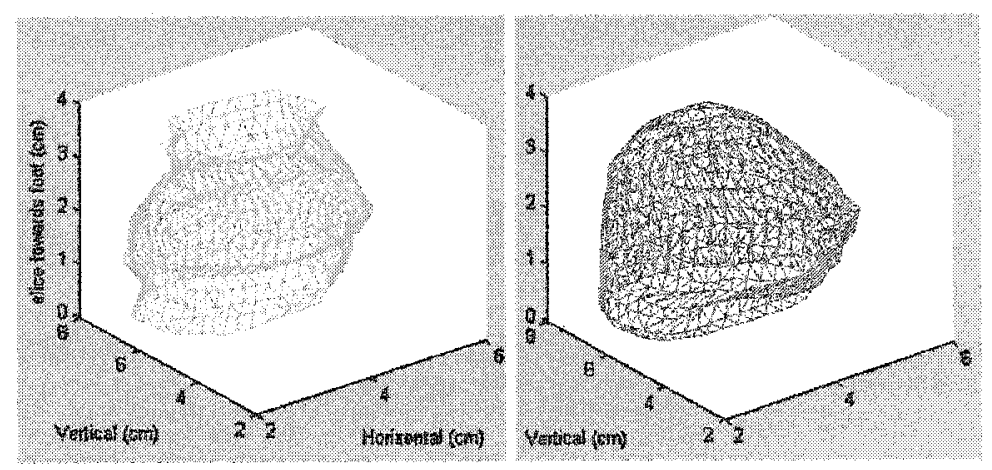

Figure 5.2: The CT (left) and the Photo (right) 3D prostate surfaces

\begin{tabular}{lrrrr|} 
CT Volume: & Min & Max & Median & STD \\
\cline { 2 - 5 } Physician1 & 25.034 & 34.872 & 29.689 & 2.503 \\
Physician2 & 19.014 & 25.257 & 20.316 & 1.648 \\
Physician3 & 28.570 & 33.582 & 30.654 & 1.277 \\
\cline { 2 - 5 }
\end{tabular}

Golth Wolturase: 20.014

Figure 5.3: The CT and the Photo volume $\left(\mathrm{cm}^{3}\right)$ computed from the definitions

\section{Prostate volume}

The prostate volume is used to describe the size of a prostate. For a man in a certain 
age range, the size of his prostate has a roughly fixed range (e.g. around $20 \mathrm{~cm}^{3}$ for a young man at the age of thirty). The definition error would be obvious if the definition volume is exceeding the fixed range. In the volume computation, the inputs are the prostate contours as defined by the three physicians at the ORCC. The CT definition volumes have been computed using our volume computation algorithm described in Section 3.4.1. Figure 5.3 shows the computation result of the volumes. The column items in the figure are the 3DSTD deviations of the CT definition volumes. The Photo definition volume is also computed and has the value of $20 \mathrm{~cm}^{3}$.

\section{Interpretations}

Considerable differences exist among the physicians and to the gold standard. The maximum volume difference represents $67.8 \%$ of the Photo definition volume, and the maximum STD is $12.5 \%$ of the Photo definition volume. The $3 \mathrm{D} \mathrm{CT}$ view in Figure 5.3 looks as if it is wearing a flat hat, compared with the smooth apex in the Photo, apparently there is no further smaller contour defined on the more inferior CT slices (toward the foot direction).

\subsubsection{CT and Photo Registration}

The registration is to register the $\mathrm{CT}$ and the Photo images into a common coordinate system, such that the error analysis can be performed to estimate our physicians' definitions on the CT images by comparing with that on the Photo images. We perform full images registration. The input to the registration program are the thirty-four axial (transverse) CT scans which cover the entire prostate at $1 \mathrm{~mm}$ intervals with the slice resolution of $512 \times 512$ pixels (pixel size: $0.89 \mathrm{~mm}$ ), and the thirty-four transverse 
Photo scans with the same slice intervals. The Photo image resolution is $1216 \times 2048$ pixels (size: $0.33 \mathrm{~mm}$ ). In the initial stage of the registration process, we perform the following operations: first we re-scale the CT slices, such that the CT slices and the Photo slices have the same resolution of $1216 \times 2048$ pixels. Secondly we map the CT's median point to the Photo's median point, and extract a same-size region ( $768 \times 1392)$ from both images for the registration, which include the outer contour of the pelvic slice. In the third operation, we mask out the Photo's background noise (not exist in the CT images) by using Matlab's edge function to obtain the Photo mask for the removal of the noise to increase the mutual information value. A graphic comparison of the before masking and the after masking is shown in Figure 5.4.
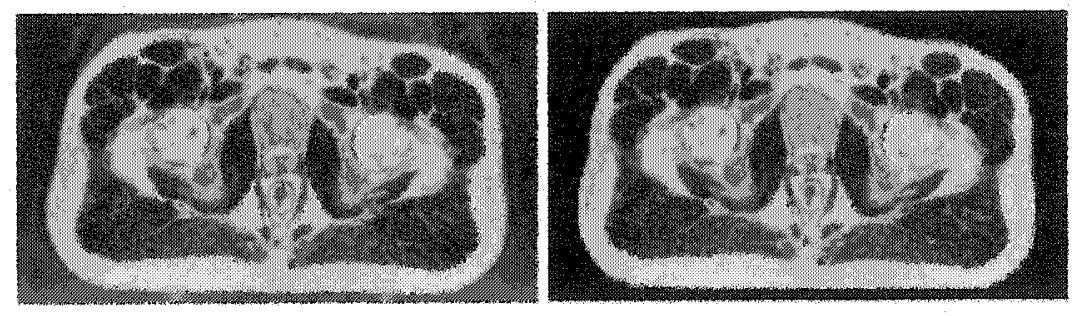

Figure 5.4: Mask out the background noise on a Photo slice. The left picture is the before masking, and the right picture is the after masking

After the initial stage, the joint histogram of the CI volume and the Photo volume is computed iteratively to obtain a set of the optimized parameters using the mutual information technique described in Section 3.3. The parameters include the three rotations and three translations in the $X, Y, Z$ axes. In the registration process, the multi-resolution technique is used to speed up the registration algorithm to find the optimum registration parameters. First we re-scale all of the CT images and Photo images such that one out of eight pixels are sampled in the $X$ and $Y$ axes for obtaining 
a lower resolution of 96 pixels by 174 pixels comparable with the original size of 768 pixels by 1392 pixels. The algorithm proceeds with the registration of the two set of the lower resolution images and obtains a set of the optimized parameters. Then the images of the higher resolution of 192 pixels by 384 pixels are obtained by the rescaling such that one out of four pixels are sampled in the $X$ and $Y$ axes of the original CT images. For the higher resolution image registration, the input are the one fourth resolution of images and the optimized parameters obtained from the lower resolution registration. Therefore we start with the lower resolution of one-eighth to compute an estimation and use it as the initial estimate for the higher resolution of one-fourth. From the experiment we observed that the result of using the lower resolution is very close to that of the high resolution. Figure 5.5 gives the registration results. We can see that the two resolution levels are enough for a good registration. There is no large difference from the level of one-eighth to that of one-fourth. There is only about one pixel difference in the $\mathrm{X}$ axis. The results coincide with the finding [14], which states that two level multi-resolution is sufficient for the image registration.

\begin{tabular}{|ccccccccc|}
\hline \multicolumn{7}{c}{ Computed registration parameters } \\
& $\mathrm{rx}$ & $\mathrm{ry}$ & $\mathrm{rz}$ & $\mathrm{tx}$ & $\mathrm{ty}$ & $\mathrm{tz}$ & size (pixel) & time (min) \\
\hline $1 / 8$ & 0.0012 & -0.0006 & -0.6066 & -2.0232 & -0.0304 & 0.0052 & $96 \times 174 \times 34$ & 10 \\
$1 / 4$ & 0.0012 & -0.0006 & -0.6066 & -1.0620 & -0.0152 & 0.0046 & $192 \times 348 \times 34$ & 3 \\
& & & & & & & & \\
\hline
\end{tabular}

Figure 5.5: The multi-resolution of $1 / 8$ and $1 / 4$ applied to the $C T$ and the Photo in the 3D rigid registration. The parameters $\mathrm{rx}, \mathrm{ry}, \mathrm{rz}$ define the rotations around $\mathrm{X}, \mathrm{Y}, \mathrm{Z}$ axes respectively; and the parameters $t x, t y, t x$ define the translation about $X, Y, Z$ axes respectively. 
We use the checker box method [14] to examine the quality of the registration. Figure 5.6 shows the result of our registration of the $\mathrm{CT}$ and Photo volumes using the checker box images. The registration in our lab takes 10 minutes for the one-eighth resolution. For the one-fourth resolution, the converging time is about 3 minutes, based on the estimated result of one-eighth resolution. The converging time of the higher resolution registration is less than that of the lower resolution. It is because the higher resolution registration starts with a good estimation of parameters computed in the lower resolution one. Only a close neighborhood of the parameters needs to be searched in the higher resolution registration, thus less iterations (less computations) are used for the final converging of the algorithm.

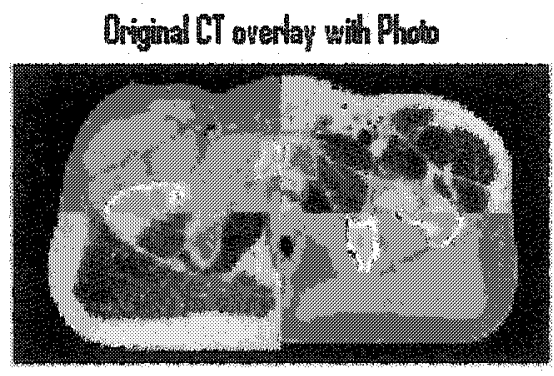

(a)

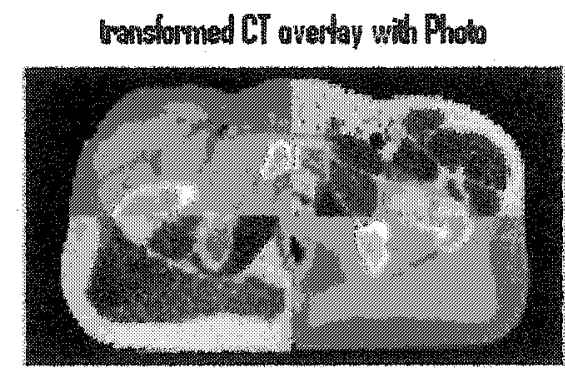

(b)

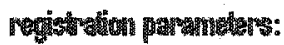

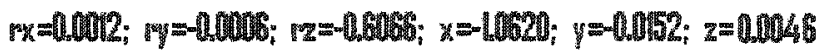

Figure 5.6: A checker-box comparison of the CT and the Photo innages before the registration and after the registration. (a) shows some misalignment in the vertical direction and horizonal direction. Whereas (b) shows that the misalignment has been corrected. The Photo image is more dark than the CT(gray color) image. 


\subsubsection{D error analysis}

A set of statistics has been designed into the prostate definition evaluation system. A user can select a physician and a slice as the input to our $2 \mathrm{D}$ analysis program. The $2 \mathrm{D}$ error analysis program includes definition contour overlay, frequency measurement, over- and under-estimations.

\section{Definition contour overlay}

We compute the minimum, maximum, median, and STD (3MSTD) contours from a physician's twenty definitions for a particular slice. The minimum measurement is to measure the worst possible under-estimation that a physician makes in defining the prostate on a slice. The maximum measurement is to measure the worst possible overestimation. Whereas the median measurement is to measure how close the prostate definition is to the Photo definition. The STD measurement is to measure the average deviations from the median measurement.

To compute the 3MSTD statistics, the user

- select a physician

- select a CT slice

The system fetches

- all (20) CT contours

- Photo contour

The 3MSTD contours are computed using the ray-shooting method described in Section 3.4.3. Then all of the contours including the computed ones (e.g., STD, median) are plotted in the same coordinate system. The buttons of $m m$ on and $m m$ of $f$ are 
designed to switch on and off the displaying of the minimum contour and the maximum contour for the viewing purpose. Whereas the buttons (to foot and to head) are designed on the window for the viewers to scroll through the CT slices, and it is more like a movie show. An overlay view is shown in Figure 5.7, with some different colors. The two sets of STD points create a buffer around the median contour to show the accuracy range of the definitions.

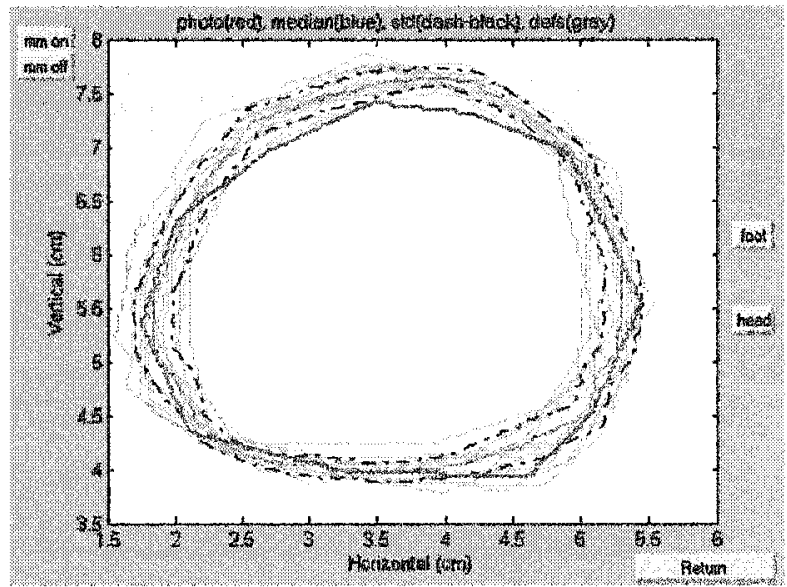

Figure 5.7: The prostate definitions (20 times) of a slice overlayed together. The red contour is the Photo definition; the gray one is the CT definition; the dashed black ones are the STD; the blue one is the median.

\section{Frequency measurement}

In our frequency statistics, we measure and illustrate how often each pixel is classified as belonging to the prostate.

To compute the statistics, the user

- select a physician

- select a CT slice 
The system fetches

- all (20) CT contours

- Photo contour

Then for each pixel of the CT slice, the counts of the pixel which is included in the contour definitions are obtained as the frequency measurement. Figure 5.7 gives the plot of the frequency distribution of the definitions. We can see that the more times the pixel is included in the target, the higher the frequency, and the brighter the color of the pixel. We super-impose the Photo definition with different color (black) onto the plot to give the over- and under-estimation locations.

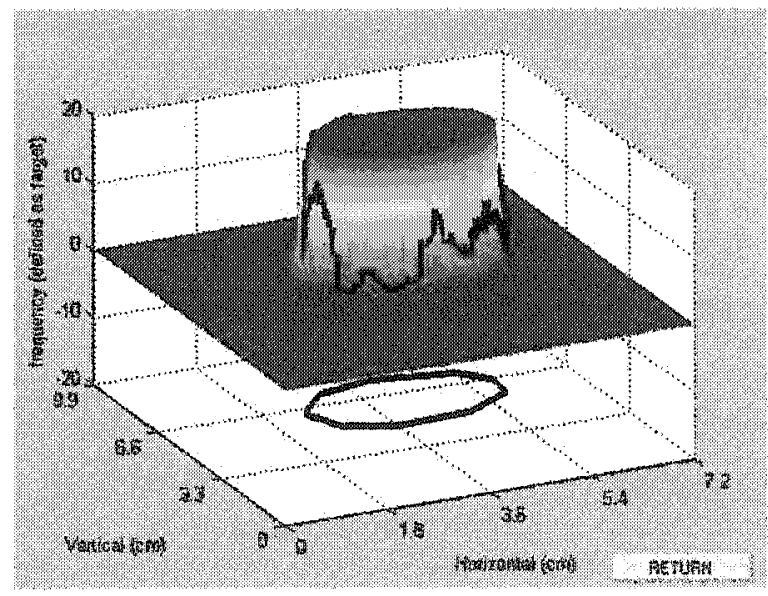

Figure 5.8: The frequency of a slice definition superimposed with that of the Ploto

In Figure 5.8, the Photo definition contour, for the same slice as in the CT, is shown on the floor plane to indicate its shape and location. We can observe that, the top side of the definitions has higher definition frequencies (where the thick black contour curves up), thus they have a higher coverage; and the bottom has lower defnition 
frequencies where the thick black contour curves down), thus lower coverage. These findings reaffirm with the findings in the published papers [23, 24].

\section{Over-and under-estimations}

The aim of these measurements is to estimate the maximum errors that the physician contributes in the target definitions in terms of the over- and under-estimations. We first overlay all of the prostate definitions on a CT slice from a physician together with the definition on the Photo slice. Then we obtain a median point (we assume it exists) which enclosed by all the overlayed definitions, and compute the maximum over- and under-estimates using the ray-shooting method described in Section 3.4.4. Figure 5.9 is the overlay of all of the target definitions on a CT slice. Figure 5.10 is the illustration of the computation with the graph (a) representing the enlarged definition area of the prostate. 


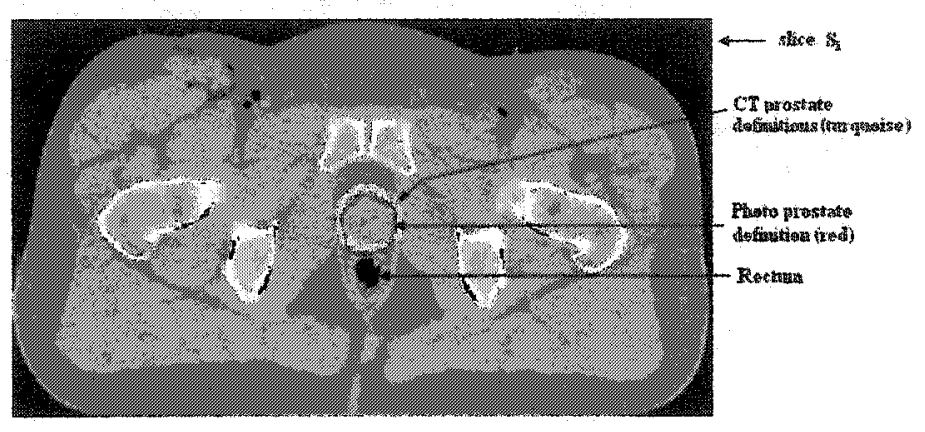

Figure 5.9: The illustration of the prostate definitions. The $20 \mathrm{CT}$ definition contours(turquoise) and 1 Photo definition(red) contour are superimposed on the anatomical slice.

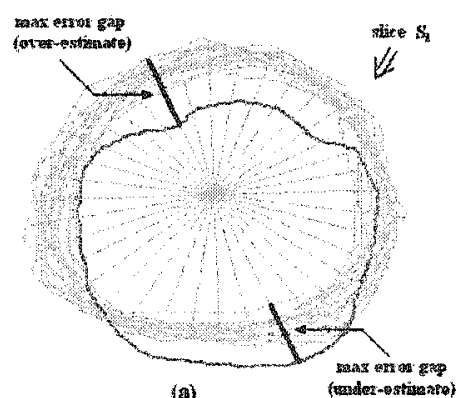

$(s)$

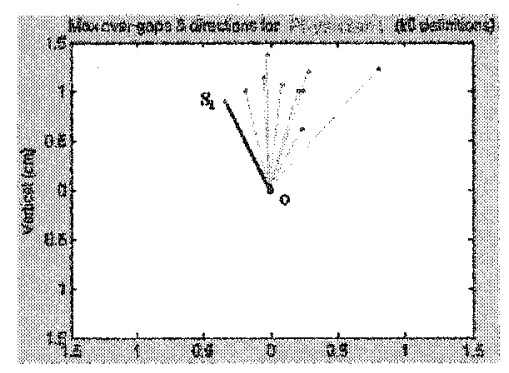

(b)

Figure 5.10: The illustration of computation of the maximum over- and under-estimation gaps and directions using the ray-shooting method. (a) is the overlays of all of the twenty prostate definitions on the CT slice $S_{i}$ in gray color and one prostate definition on the Photo slice in red color. The maximum error gaps of the over- and under-estimates are shown in black thick lines respectively. (b) is an accumulated error gap plot of all of the prostate slices in a polar coordinate system originated at the black point o; 
In (b) (Figure 5.10), each of the blue dots presents a slice; the line segment connecting a blue dot and $o$ presents the maximum over-estimated gap. The segment direction indicates the error direction. The segment which has its blue dot labeled $S_{i}$ corresponds to the maximum over-estimated gap for the slice $S_{i}$ in $(a)$.

The statistics of the maximum over- and under-estimates of all of the three physicians are computed this way and shown in Figure 5.11. The top two graphs of the figure are for the physician1; the middle two are for the physician2, and the bottom two are for the physician3.

\section{Interpretations}

As for the in-slice definition errors, the over-estimates mostly occur at the top direction (anterior) and appear more scattered. Whereas the under-estimates mostly occur at the bottom direction (posterior) and appear more clustered. The maximum error gap (length) is about $25 \%$ of the prostate diameter $(6 \mathrm{~mm})$. The reason for the under-estimates at the posterior direction, as explained by our physicians, is that the physicians tend to avoid including the rectum due to its extremely sensitive to radiation during subsequent treatment, when they cannot tell the prostate extent at the posterior. Also there are the tendencies to include the area as much as possible at the anterior because there are no critical structure above the prostate, thus causing the over-estimations. We can have the conjecture that the median definition can be considered as the 'quick and dirty' method to reduce the definition variations. 


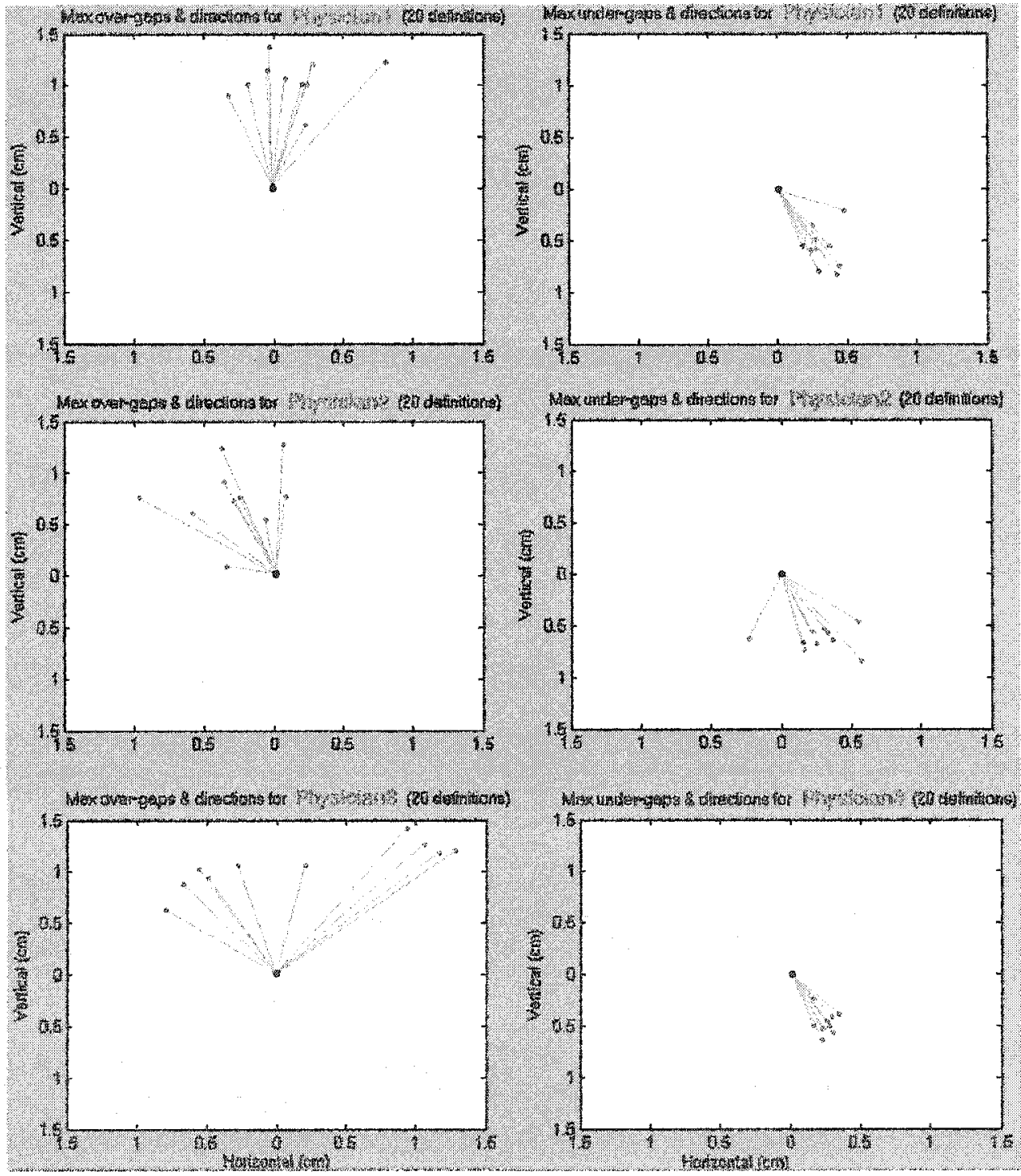

Figure 5.11: The maximum over- and under-estimate gaps and directions 


\subsubsection{D error analysis}

This section includes the $3 \mathrm{D}$ view of the definition errors and the Oblique presentation of the transverse CT definitions.

\section{$3 D$ view of the definition errors}

The 3D volume analysis and statistics computation are performed in our experiments. We have computed the 3MSTD volumes for visualizing the CT volume together with the Photo volume. The construction of these images give the $3 \mathrm{D}$ views of the worst possible prostate definition errors of the physicians. In the beginning of the computation process, the user selects a physician and all of the prostate definitions of the physician are loaded into the memory. Then all of the definitions of a physician on a slice are overlaid and the ray-intersect technique is applied to compute minimum, maximum and median contours, which are then used for the 3D reconstruction. Figure 5.12 gives $3 \mathrm{D}$ views of minimum, maximum CT definitions superimposed with that of the Photo.

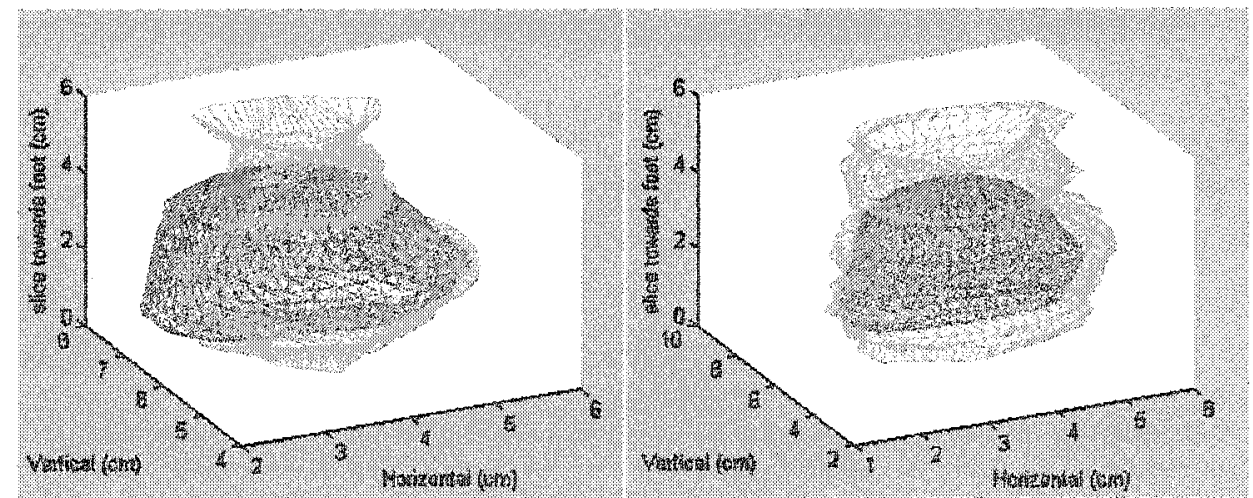

Figure 5.12: The left graph is the minimum CT definition volume superimposed with the Photo volume; the right graph is the maximum crr definition volume superimposed with the Photo volume. 


\section{Volume statistics}

Figure 5.13 shows a table of the statistics for the coverage, the over- and the underestimates of the three physicians. The maximum prostate volume coverage is $88.51 \%$, and the maximum over-estimate is $83.32 \%$.

\begin{tabular}{|c|c|c|c|c|c|c|}
\hline \multirow[b]{2}{*}{ Physician1 } & \multicolumn{2}{|c|}{ Coverage } & \multicolumn{2}{|c|}{ Over-estimate } & \multicolumn{2}{|c|}{ Under-estimate } \\
\hline & $\mathrm{cm}^{3}$ & $\%$ & $\mathrm{~cm}^{3}$ & $\%$ & $\mathrm{~cm}^{3}$ & $\%$ \\
\hline $\min$ & 16.77 & 83.79 & 7.72 & 38.57 & 1.73 & 8.66 \\
\hline $\max$ & 18.28 & 91.34 & 16.68 & 83.32 & 3.25 & 16.21 \\
\hline median & 17.54 & 87.64 & 12.15 & 60.70 & 2.47 & 12.36 \\
\hline std & 0.40 & 1.99 & 2.37 & 11.85 & 0.40 & 1.99 \\
\hline \multicolumn{7}{|l|}{ Physician2 } \\
\hline $\min$ & 14.55 & 72.72 & 3.51 & 17.53 & 2.30 & 11.49 \\
\hline $\max$ & 17.72 & 88.51 & 8.02 & 40.07 & 5.46 & 27.28 \\
\hline median & 15.70 & 78.45 & 4.94 & 24.68 & 4.24 & 21.18 \\
\hline std & 0.86 & 4.29 & 1.06 & 5.31 & 0.86 & 4.29 \\
\hline
\end{tabular}

Physician3
\begin{tabular}{l|rr|rr|rr|}
$\min$ & 16.02 & 80.07 & 11.98 & 59.86 & 2.62 & 13.10 \\
$\max$ & 17.39 & 86.39 & 16.28 & 81.34 & 3.99 & 19.93 \\
median & 16.69 & 83.39 & 13.42 & 67.05 & 3.03 & 15.13 \\
std & 0.33 & 1.64 & 1.02 & 5.12 & 0.33 & 1.64 \\
\hline
\end{tabular}

Gold Volume: 20.01

Figure 5.13: The CT definition volume statistics, $\%$ - a measurement divided by the Photo volume.

\section{Interpretations}

We can see that there are considerable definition errors revealed in the 3D view and the volume statistics. In the slice direction, the physicians tend to include more slices in the inferior and the superior directions due to the poor CT quality in locating the base and apex of the prostate. From the definition data, we see that all of the three physicians had either missed or over-defined at least two slices, and each slice is $3 \mathrm{~mm}$ thick. The visual effect of the over-estimations in the slice direction can be seen in 
Figure 5.12. From our volume coverage, we see that maximum median coverage is only $87.6 \%$ of the three physicians, and the inter-physician variations appear quite large (std: $11.8 \%$ vs. $5.1 \%$ ). The impact is that large amount of healthy tissues specically the rectum and the bladder, which are extremely sensitive to radiation, are at the risk of being radiated during raditation treament and thus causing bleeding and great pains in the patient.

\subsubsection{Oblique presentation of definitions}

Figure 5.14 gives a snapshot of the oblique presentation of the transverse contour definition in the evaluation system. The two oblique slices are displayed along with the transverse slice which is located in the center of the window screen. A dummy figure is located above the transverse window and its three lines indicate the anatomic locations of the current transverse and oblique slices. The blue dots above the rectum in the oblique slices are the intersection points of the definition. With the help of the oblique presentation, the physician can determine whether the current transverse Contour Definition is correct or not. Because the additional bony structures revealed in the oblique slices, which can not be seen in the transverse slice.

\subsection{Contouring system}

Some experimental work has been conducted on our contouring system which aims to help the physicians in specifying the prostate target. This section gives the descriptions of the system operation, the three window contouring, the problem encountered 


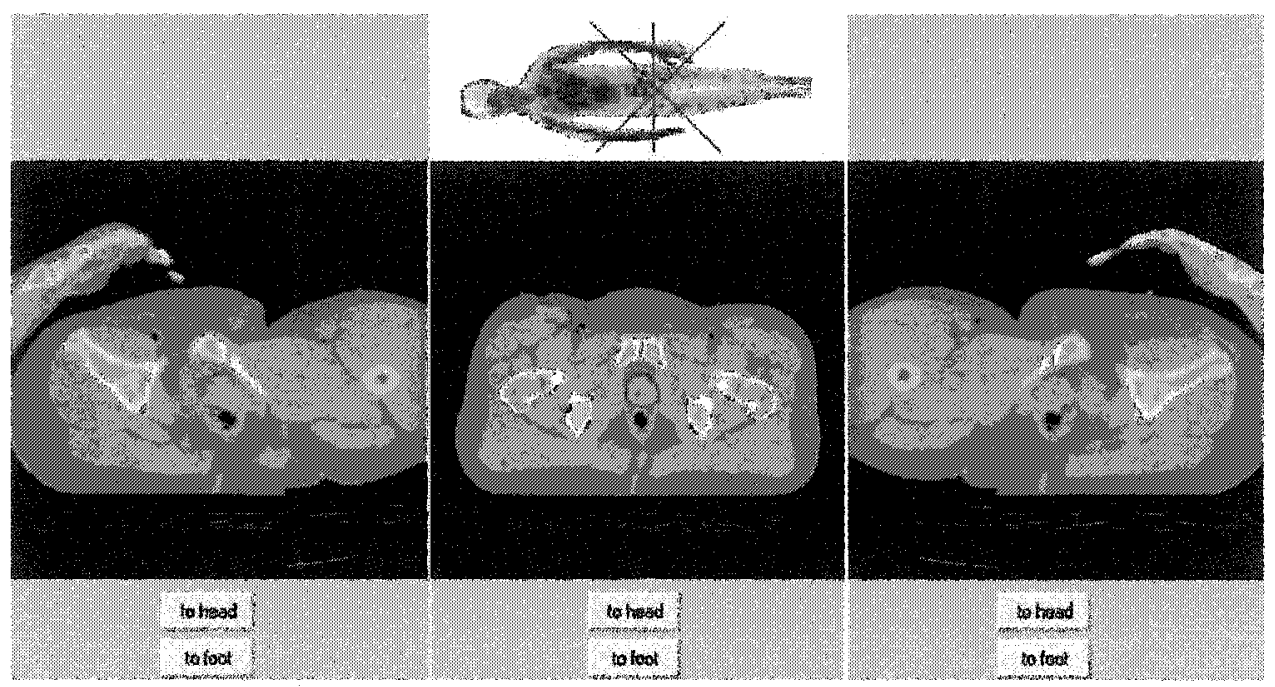

Figure 5.14: The Oblique presentation of definitions.

in the oblique slice computation.

\subsubsection{System operation}

Figure 5.15 gives the screen flow diagram to show the hierarchy flow control of the contouring system. The description of the system operation is given below.

The user starts the systems by executing the main program EvalDemo.m in Matlab and the main menu (a) of Evaluation and Contouring appears (Figure 5.15). There are two system options on menu (a) Definition Evaluation and Prostate Contouring. The user clicks on the Prostate Contouring option to start the contouring system and the Prostate Contouring menu (b) appears. Menu (b) has one option available: Oblique Contouring(-deg). The other options are dimmed (not implemented in this 
study). The available option enables the user to manually draw target contours on the right oblique window, and presents the drawn contours on the transverse window and the left oblique window. When the user clicks on the available option on menu (b), the Contouring on Right Oblique(-deg) menu (c) appears. The user clicks on the Do Contouring to start the contouring process. At the beginning of the contouring process, the $\mathrm{CT}$ anatomical slices are loaded into the memory and two sets of the oblique slices (left oblique and right oblique) are computed using the method described in Section 3.6.1. The following section presents the three window contouring.

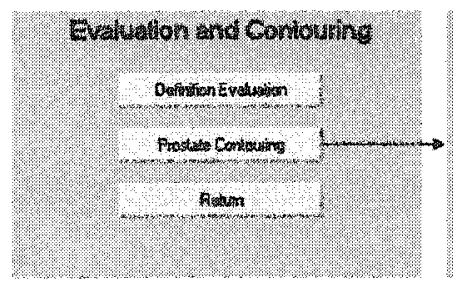

(a)

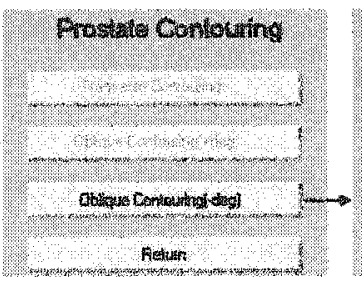

(b)

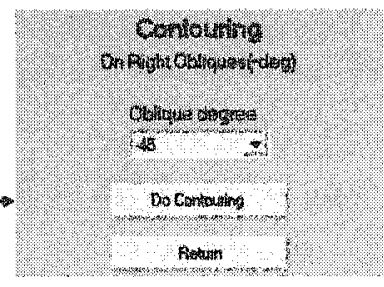

(c)

Figure 5.15: The control flow of the contouring system. The dimmed buttons indicate that the modules have not implemented in this study.

\subsubsection{The three window contouring}

The Figure 5.16 shows the transverse slice (middle), the 45 degree oblique slice (left) and the -45 degree oblique slice (right). We start drawing the contours on the -45 degree oblique slice. There are some push buttons located on the top of the right oblique view to help the users in the contouring. The user can click on the Contouring button to start drawing a contour on the current slice, and the user can hit enter 
when finish. The Clear contour button can be clicked if the user is not satisfied with the finished contour. The Done button is to compute the intersection points of the current finished contour with the other two views which are located on the left and the center of the screen. The computed points will be shown on these two views if they do intersect. The user can flip through the different slices of the two views and observe the position changes of the computed intersecting points. The distinct bone structures shown on the oblique views will help physicians make a decision as to where the contour would be when the tissues' contrast is not very clear on one of the views. There are also two editing buttons for the users to do some contour editing. The users can choose two points on the current contour and the shorter segment between the 2 points will be removed. Then the user can add some new points in between the two points as the replacement of the removed ones, and they hit ENTER when finish. On top of the transverse view, there is a dummy figure with three lines to indicate the slice positions.

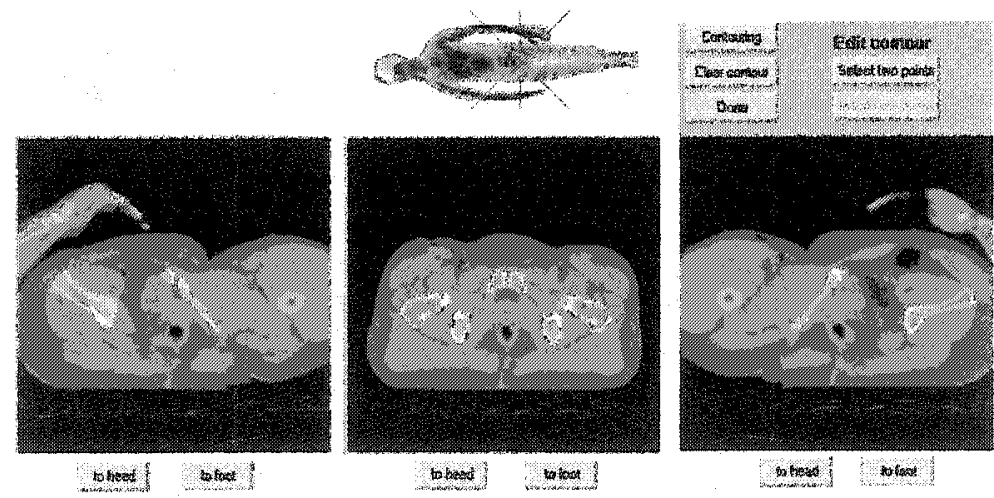

Figure 5.16: Three window contouring tool 


\subsubsection{Issue in computing oblique slices}

\section{Image contrast problem}

We compute a few sets of oblique slices, using the interpolation and the transformation methods described in Chapter 3. The contrast of the oblique slices does not look uniformed in some part of the slices, part of the left side is brighter than that on the right. This is due to the different brightness with some transverse slices. Therefore we did some brightness adjustments manually to the problematic slices of the transverse, by using Photoshop. The image contrast is adjusted using histogram values and an automatic procedure can be developed to adjust the images to an unformed brightness desired. A comparison of the pre- and post-adjustments are shown in Figure 5.17:
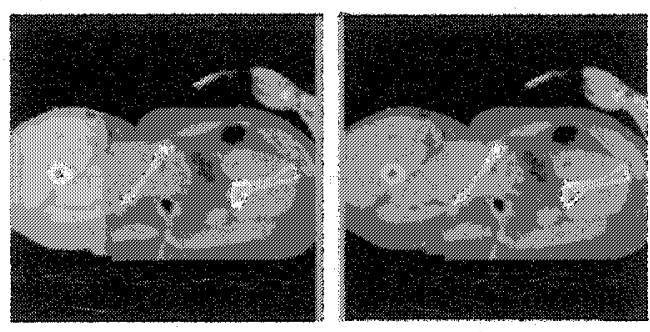

Figure 5.17: The oblique slice on the left is before the brightness adjustments and the one on the right is after the brightness adjustments. 


\section{Chapter 6}

\section{Conclusion}

We have developed a system for the evaluation of the quality of the contouring by a physician and a system for enhanced contouring. During the target contouring, a physician may face the problem of poor image quality due to the CT's low soft-tissue contrast. The evaluation system can assist the physicians in the error analysis of their target and organ contour definitions in 2D and 3D. In the evaluation system, we use CT and Photo images of the prostate as the input to the system. The target definitions on the Photo images are taken as the gold standard to compare with those on the CT images, because the Photo images provide more realistic and more accurate comparative references than the conventional gold standard images (CT). We developed a new fast 3D volume computation algorithm which is very accurate compared with the estimated methods in literature. Based on our experiments, the volume accuracy is improved by about $15 \%$ using our method. Our system produces the visual images of the definitions in $2 \mathrm{D}$ and $3 \mathrm{D}$, computes a set of the evaluation statistics, and presents the definitions on the computed oblique slices which reveal 
some additional structures surrounding the target. For example, the experimental results of our system showed that the definitions of the prostate extent on the conventional transverse CT images by the various experienced physicians contribute to large under-and over-estimations. Considerable errors exist in the physicians' target definitions in terms of the prostate volume coverage, the error gaps and the direction tendencies.

These definition errors are unavoidable due to the CT's poor image quality. Therefore we developed our new 3D contouring tool to aid in the definition task and reduce definition errors. Our novel approach aids to dynamically reveal 3D structures of the targets, which is not possible in the traditional 2D contouring setting. Using our contouring system, users can manually draw contours on the target slices by computer mouse and the drawn contours can be easily edited or cleared. Three separate windows are designed in the system: one for transverse slices, one for left oblique slices and one for right oblique slices. Users can define the target in any of the windows and the drawn target contours can be presented on the other two windows for the cross-references. Our new approach has received a great interest from the physicians who are involved in the evaluation work, and it potentially can be integrated into the current clinical application.

\section{Future work}

Currently our oblique CT slices are pre-computed due to the large computation time requirement, which is in minutes. This needs to be solved before the tool can be applied in the clinical setting. An approach that we are considering is to compute only the region of interest (ROI) around the relevant surroundings of the prostate, and superimpose the ROI with an oblique template to reveal the rest of the anatomic 
structures. The ROI on a slice should include the critical structures, which surround the targets (i.e., the rectum, the bladder). In this way, the computation of an oblique slice could be reduced to an area of $8 \mathrm{~cm}$ by $8 \mathrm{~cm}$ (equivalent to $80 \times 80$ pixels), which is significantly smaller than a whole CT image of size of $512 \times 512$. With today's computing speed, this computation could be done within a few seconds.

We are using Matlab as the platform for our contouring system. Matlab is an interpreter language. It has a large number of powerful library functions (e.g., optimizations, matrix manipulations) and GUI for fast system development. On the other hand, it is slow comparing to other programming languages, such as $\mathrm{C} / \mathrm{C}++$. To increase the application speed, it is desirable to implement the tool in the $\mathrm{C} / \mathrm{C}++$ like languages. 


\section{Bibliography}

[1] H.N. Christiansen and T.W. Sederberg, Conversion of complex contour line definitions into polygonal element mosaics, ACM SIGGRAPH Computer Graphics, 12(3):187-92, August 1978

[2] Ge Cong, Bahram Parvin, Robust and efficient surface reconstruction from contours, The Visual Computer, 17(4): 199-208, 2001

[3]. James V. Miller,David E. Breen,William E. Lorensen, Robert M. O'Bara, Michael J. Wozny, Geometrically deformed models: a method for extracting closed geometric models form volume data, Computer Graphics, 25:217-226, 1991

[4] Lorensen, William and Harvey E. Cline, Marching cubes: A high resolution 3D surface construction algorithm, Computer Graphics, SIGGRAPH 87 Proceedings, 21(4):163-170, Jul. 1987

[5] J. Jeoung, K. Kim, H. Park, H. Cho, and M. Jung, B-spline surface approximation to cross-sections using distance maps, International Journal of Advanced Manufacturing Technology, 15: 876-885, 1999 
[6] Fuji Kikuo, K. Eddy, Shape reconstruction from contours using isotopic deformation, Graphical models and image processing: GMIP, 61(3):127-147, 1999

[7] Hown-Wen Chen and Ju-Rone Ho, A nonlinear technique for reconstruction of 3D medical images, CVGIP: Graphical Models and Image Processing, 53(4):382391, July 1991

[8] C. Coleman, Michael D. Roberts, Christian M.,Maender and Richard T. Hart, Solid model creation from CT image data - technical report, Bone Tissue Mechanics Lab, Tulane University, Mar. 2000

[9] A. Zizzari, B. Michaelis and G. Gademann, Simulation and modeling of brain tumors in computer-assisted radiotherapy, Proceedings of the IASTED International Conference on Applied Simulation and Modeling, ASM), Marbella, Spain, September 3-5, 2003

[10] Studholme C, Hill DL, Hawkes DJ, Automated three-dimensional registration of magnetic resonance and positron emission tomography brain images by multiresolution optimization of voxel similarity measures, Medical Physics, 24(1):25-35, Jan. 1997

[11] Studholme C, Hill DL, Hawkes DJ, An Overlap invariant entropy measure of 3D medical image alignment, Pattern Recognition, 32(1): 71-86, Dec 1998

[12] Collignon A, Maes F, Delaere D, Vandermeulen D, Suetens P, Marchal G., Automated multimodality image registration using information theory, in Proceedings of Information Processing in Medical Imaging, 263-274, 1995 
[13] Maes F, Collignon A, Vandermeulen D, Marchal G, Suetens P., Multimodality image registration by maximization of mutual information, IEEE Transactions of the Medical Imaging, 16(2):187-98, Apr. 1997

[14] Maes F, Vandermeulen D, Suetens P., Comparative evaluation of multiresolution optimization strategies for multimodality image registration by maximization of mutual information, Medical Image Analysis, 3(4):373-86, Dec. 1999

[15] Wells WM 3rd, Viola P, Atsumi H, Nakajima S, Kikinis R., Multi-modal volume registration by maximization of mutual information, Medical Image Analysis, 1(1):35-51, Mar. 1996

[16] Woods RP, Mazziotta JC, Cherry SR., MRI-PET registration with automated algorithm, Journal of Computer Assisted Tomography, 17:536-546, 1993

[17] Maintz JB, Viergever MA., A survey of medical image registration, Medical Image Analysis, 2(1):1-36, Mar. 1998

[18] William T. Press, Saul A. Teukolsky, William T. Vetterling, Brian P. Flannery, Numerical recipes in C, Cambridge university press, 1988

[19] K. Kuczynski and P. Mikolajczak, Information theory based medical images processing, Opto-Electronics Review, 11(3):235-259, 2003

[20] Zhu YM., Volume image registration by cross-entropy optimization, IEEE Transactions of the Medical Imaging, 21(2):174-80, Feb. 2002

[21] Michael J. Ackerman, Accessing the Visible Human Project, D-Lib Magazine, 1995 
[22] Eds. I. S. Evans, R. Dikau, E. Tokunaga, H. Ohmori and M. Hirano, , Multiresolution spline models and their applications in geomorphology, Concepts and modeling in Geomorphology: International Perspectives, TERRAPUB, Tokyo, 221-237, 2003

[23] Fiorino C, Reni M, Bolognesi A, Cattaneo GM, Calandrino R., Intra- and interobserver variability in contouring prostate and seminal vesicles: implications for conformal treatment planning, Radiation Oncology, 47(3):285-92, Jun. 1998

[24] Zelefsky MJ, Crean D, Mageras GS, Lyass O, Happersett L, Ling CC, Leibel SA, Fuks Z, Bull S, Kooy HM, van Herk M, Kutcher GJ., Quantification and predictors of prostate position variability in 50 patients evaluated with multiple CT scans during conformal radiotherapy, Radiation Oncology, 50(2):225-34, Feb. 1999

[25] Logue JP, Sharrock CL, Cowan RA, Read G, Marrs J, Mott D., Clinical variability of target volume description in conformal radiotherapy planning, International Journal of Radiation Oncology Biology Physics, 41(4):929-31, Jul. 1998

[26] Rasch C, Barillot I, Remeijer P, Touw A, van Herk M, Lebesque JV., Definition of the prostate in CT and MRI: a multi-observer study, International Journal of Radiation Oncology Biology Physics ., 43(1):57-66, Jan. 1999

[27] Lee WR, Roach M 3rd, Michalski J, Moran B, Beyer D., Interobserver variability leads to significant differences in quantifiers of prostate implant adequacy, International Journal of Radiation Oncology Biology Physics ., 54(2):457-61, Jan. 2002 
[28] Leunens G, Menten J, Weltens C, Verstraete J, van der Schueren E., Quality assessment of medical decision making in radiation oncology: variability in target volume delineation for brain tumours, Radiation Oncology, 29(2):169-75, Nov. 1993

[29] Tai P, Van Dyk J, Yu E, Battista J, Stitt L, Coad T., Variability of target volume delineation in cervical esophageal cancer, International Journal of Radiation Oncology Biology Physics . , 1;42(2):277-88, Sep. 1998

[30] Cazzaniga LF, Marinoni MA, Bossi A, Bianchi E, Cagna E, Cosentino D, Scandolaro L, Valli M, Frigerio M. Interphysician variability in defining the planning target volume in the irradiation of prostate and seminal vesicles, Radiation Oncology, 47(3):293-6, Jun. 1998

[31] Ketting CH, Austin-Seymour M, Kalet I, Unger J, Hummel S, Jacky J., Consistency of three-dimensional planning target volumes across physicians and institutions, International Journal of Radiation Oncology Biology Physics, 37(2):445-53, Jan. 1997

[32] William Kahan, What has the volume of a tetrahedron to do with computer programming languages, Seminar on Computational Linear Algebra http://www.cs.berkeley.edu/wkahan/VtetLang.pdf, Apr. 2001

[33] Paul Bourke, Calculating the area and centroid of a polygon, http://astronomy.swin.edu.au/pbourke/geometry/polyarea/, Jul. 1988

[34] Uche Akotaobi, Points, planes, and vector math, CECS, University of Southern California; http://www-scf.usc.edu/akotaobi/gptut11.html, Nov. 2002 
[35] Mathworks, Matlab, http://www.mathworks.com/, 1994-2004

[36] Tai P, Van Dyk J, Yu E, Battista J, Stitt L, Coad T., Variability of target volume delineation in cervical esophageal cancer, International Journal of Radiation Oncology Biology Physics . , 42(2):277-88, Sep. 1998

[37] Bjorn Hamre, 3D image registration of magnetic resonance (MRI) head volumes. Cand. scient. thesis, Section for Medical Image Analysis and Informatics, Department of Physiology \& Department of Informatics; University of Bergen, Norway, Dec. 1998

[38] Dr. Lori Scarlatos, computer graphics - course notes, http://acc6.its.brooklyn.cuny.edu/lscarlat/graphics/transform.html, 2000

[39] Salmon, R., \& Slater, M., Computer graphics, Systems and Concepts (Reading, MA: Addison-Wesley), 1987

[40] Mohan R, Barest G, Brewster LJ, Chui CS, Kutcher GJ, Laughlin JS, Fuks Z., A comprehensive three-dimensional radiation treatment planning system, International Journal of Radiation Oncology Biology Physics, 15(2):481-95, Aug. 1988

[41] Goitein M, Abrams M., Multi-dimensional treatment planning: I. Delineation of anatomy, International Journal of Radiation Oncology Biology Physics, 9(6):77787, Jun. 1983 